\title{
COMPACT IMBEDDINGS IN ELECTROMAGNETISM WITH INTERFACES BETWEEN CLASSICAL MATERIALS AND META-MATERIALS
}

\author{
LUCAS CHESNEL* AND PATRICK CIARLET JR. ${ }^{\dagger}$
}

\begin{abstract}
In a meta-material, the electric permittivity and/or the magnetic permeability can be negative in given frequency ranges. We investigate the solution of the time-harmonic Maxwell equations in a composite material, made up of classical materials, and meta-materials with negative electric permittivity, in a two-dimensional bounded domain $\Omega$. We study the imbedding of the space of electric fields into $L^{2}(\Omega)^{2}$. In particular, we extend the famous result of Weber, proving that it is compact. This result is obtained by studying the regularity of the fields. We first isolate their most singular part, using a decomposition à la Birman-Solomyak. With the help of the Mellin transform, we prove that this singular part belongs to $H^{s}(\Omega)^{2}$, for some $s>0$. Finally, we show that the compact imbedding result holds as soon as no ratio of permittivities between two adjacent materials is equal to -1 .
\end{abstract}

Key words. Maxwell's equations, interface problem, meta-material, compact imbedding, regularity of fields, Mellin transform.

AMS subject classifications.

1. Introduction. We consider the solution of the time-harmonic Maxwell equations in a composite material. A composite material is modelled by non constant electric permittivity $\varepsilon$ and magnetic permeability $\mu$. The variations of $\varepsilon$ and $\mu$ can be smooth, or piecewise smooth. Recently, some new composites appeared, including classical materials and meta-materials. A meta-material exhibits special properties. In given frequency ranges, it can behave like a material with negative electric permittivity or/and negative magnetic permeability. Examples of meta-materials $[25,26,27,12]$ include superconductors, left-handed materials, etc. Due to the sign change between a classical material and a meta-material, the usual mathematical approaches fail to resolve the corresponding electromagnetic models. In other words, these composites raise challenging questions, both from the mathematical and numerical points of view.

In this paper, we focus on an essential tool to study time-harmonic Maxwell equations in a bounded (connected) domain $\Omega$ of $\mathbb{R}^{d}$ ( $d$ is the space dimension), that is the compact imbedding of the space of electric fields in $L^{2}(\Omega)^{d}$. This result is indeed a key ingredient to solve the two instances of time-harmonic equations, namely the source problem (sustained vibrations) and the eigenvalue problem (free vibrations).

If the domain of interest is surrounded by a perfect conductor, the following functional space for electric fields, $\boldsymbol{X}_{N}(\Omega, \varepsilon)$, appears. It is made up of vector fields $\boldsymbol{v}$ that belong to $L^{2}(\Omega)^{d}$, and such that $\operatorname{curl} \boldsymbol{v} \in L^{2}(\Omega)^{d}$, $\operatorname{div}(\varepsilon \boldsymbol{v}) \in L^{2}(\Omega)$ and $\boldsymbol{v} \times \boldsymbol{n}=0$ on $\partial \Omega$, where $\boldsymbol{n}$ is the unit outward normal vector to $\partial \Omega$.

Our main objective is to find an extension of the Weber compact imbedding theorem in the case of a composite material including classical and negative metamaterials. In the landmark paper [28], Weber proved that $\boldsymbol{X}_{N}(\Omega, \varepsilon)$ is compactly

\footnotetext{
*Laboratoire POEMS, UMR 7231 CNRS/ENSTA/INRIA, ENSTA ParisTech, 32, boulevard Victor, 75739 Paris Cedex 15, France; email: lucas.chesnel@ensta.fr

${ }^{\dagger}$ Laboratoire POEMS, UMR 7231 CNRS/ENSTA/INRIA, ENSTA ParisTech, 32, boulevard Victor, 75739 Paris Cedex 15, France; email: patrick.ciarlet@ensta.fr
} 
imbedded into $L^{2}(\Omega)^{d}$ when $\varepsilon$ is a real function, bounded above and below by strictly positive constants, for $d=2,3$.

In [9], the second author and co-workers prove this result for $d=3$, in the following setting. The domain $\Omega$ is partitioned into two subdomains $\Omega_{1}$ and $\Omega_{2}$, with a smooth interface (between the subdomains), and with $\varepsilon$ exhibiting a sign-change at the crossing of the interface. This new result is proved under the assumption that the contrast $\kappa_{\varepsilon}$ (which is just $\kappa_{\varepsilon}=\varepsilon_{\mid \Omega_{1}} / \varepsilon_{\mid \Omega_{2}}$ when $\varepsilon$ is constant in each subdomains) is such that $\left.\kappa_{\varepsilon} \notin\right] \kappa_{\varepsilon}^{i} ; \kappa_{\varepsilon}^{s}[$, with $-1 \in] \kappa_{\varepsilon}^{i} ; \kappa_{\varepsilon}^{s}$. The proof is direct, in the sense that the classical result stating that the imbeddings of $H^{s}(\Omega)$ into $L^{2}(\Omega)$ are compact for $s>0$, is not used. Our method of proof, on the other hand, is to study the a priori regularity of elements of $\boldsymbol{X}_{N}(\Omega, \varepsilon)$. In this respect, it follows what has been done in proposition 3.7 of [1], or section 3 of [14], or in part 3.8 of [23], but it relies on different mathematical tools. More precisely, we shall prove that the space of electric field $\boldsymbol{X}_{N}(\Omega, \varepsilon)$ is (continuously) imbedded into spaces like $H^{s}(\Omega)^{d}$, with some ad hoc $s>0$ with the help of the Mellin transform, so that one can obtain the desired compactness result by using the classical result mentioned above. Along the way, we shall use several results of [16].

This compactness result is one of the two key ingredient result to prove that the source problem is well-posed, within the Fredholm, or isomorphism+compact, framework. The $T$-coercivity method (see [10]), or the three-field variational formulation (see [9]), then help obtaining the second key ingredient, coerciveness, so well-posedness follows. These two techniques provide some guidelines as to how the problem can be discretized.

In this paper, we shall study the two-dimensional case $(d=2)$. To simplify the proofs, we assume that the domain $\Omega$ has a connected boundary $\partial \Omega$, and that $\varepsilon$ is piecewise constant. If that were not the case, the regularity results of elements of $\boldsymbol{X}_{N}(\Omega, \varepsilon)$ should remain valid, under reasonable assumptions, such as a piecewise smooth $\varepsilon$, with $|\varepsilon|$ bounded above and below by strictly positive constants, or such as a multiply-connected boundary, which adds a finite dimensional vector subspace of curl-free and divergence-free elements of $\boldsymbol{X}_{N}(\Omega, \varepsilon)$, whose elements can be studied separately.

We begin by the notations in section 2 . We shall consider the case of a polygonal domain $\Omega$, partitioned into polygonal subdomains. In the next section, we prove a continuous splitting result for elements of $\boldsymbol{X}_{N}(\Omega, \varepsilon)$, which allows us to isolate the most singular part, expressed as a gradient of a scalar field. Then, in section 4, we study the regularity of this singular part, which leads to the compact imbedding result $(\S 5)$. We generalize this result in section 6 by assuming that the domain and subdomains are curvilinear polygons. We conclude by providing a counter-example for a symmetric cavity composed of two materials whose permittivities are opposite.

2. Notations. Let $\Omega$ be a bounded, open, connected polygonal set of $\mathbb{R}^{2}$, with connected boundary $\partial \Omega$. The unit outward normal vector to $\partial \Omega$ is denoted by $\boldsymbol{n}$, whereas $\tau$ is a unit tangent vector to $\partial \Omega$.

We assume that $\Omega$ is partitioned into $N$ open, polygonal subsets $\Omega_{j}$ :

$$
\bar{\Omega}=\bigcup_{j=1}^{N} \overline{\Omega_{j}} \text { with } \Omega_{i} \cap \Omega_{j}=\emptyset \text { if } i \neq j .
$$


We call $\mathcal{P}$ the partition. The interface between subdomains $\Sigma:=\cup_{i \neq j}\left(\partial \Omega_{i} \cap \partial \Omega_{j}\right)$ is made up of straight edges. Without loss of generality, we consider convex polygons $\Omega_{j}$, as they can be further subdivided into convex polygons if necessary. Then, to avoid unnecessary technical difficulties, we assume that the $N$ polygons of the partition $\mathcal{P}$ can be labelled so that $\overline{\Omega_{j}}$ and $\overline{\Omega_{j+1}}$ share at least one common edge, for $j=1 . . N-1$. For $j=1 . . N,\left(\partial \Omega_{j}^{k}\right)_{k=1 . . n_{j}}$ denote the set of edges of $\Omega_{j}$, and $\left(\boldsymbol{n}_{j}^{k}\right)_{k=1 . . n_{j}}$ are the corresponding unit outward normal vectors.

Let $\mathcal{A}$ denote the set of edges on the boundary $\partial \Omega$ (a side of $\partial \Omega$ can be divided into two or more edges, due to the partition), or on the interface $\Sigma$ : define $\mathcal{A}_{\text {ext }}:=$ $\{A \in \mathcal{A} \mid A \subset \partial \Omega\}$ the set of exterior edges, and $\mathcal{A}_{\text {int }}:=\mathcal{A} \backslash \mathcal{A}_{\text {ext }}$ the set of interior edges. Similarly, let $\mathcal{S}$ denote the set of vertices (new vertices can appear on $\partial \Omega$, due to the partition), and $\mathcal{S}_{\text {ext }}:=\{S \in \mathcal{S} \mid S \in \partial \Omega\}$ the set of exterior vertices, $\mathcal{S}_{\text {int }}:=\mathcal{S} \backslash \mathcal{S}_{\text {ext }}$ the set of interior vertices.

In what follows, the characteristic quantities $\varepsilon$ and $\mu$ defining the material, functions, etc. are complex valued. Let us consider more precisely $\varepsilon \in L^{\infty}(\Omega)$, constant over each subdomain $\Omega_{j}$, with $\varepsilon_{j}:=\left.\varepsilon\right|_{\Omega_{j}} \neq 0$. As a particular case, $\varepsilon$ can be real valued and exhibit a sign-shift between two neighboring subdomains. For $a<b$, we define the closed strip of the complex plane $\mathcal{B}(a ; b):=\{\lambda \in \mathbb{C} \mid a \leq \Re(\lambda) \leq b\}$.

Using differentiation in the sense of distributions, one classically defines the operators curl and div acting over $\boldsymbol{L}^{2}(\Omega):=L^{2}(\Omega) \times L^{2}(\Omega)$, and the operator curl acting over $L^{2}(\Omega)$. For $\boldsymbol{v}=\left(v_{x}, v_{y}\right) \in \boldsymbol{L}^{2}(\Omega)$,

$$
\operatorname{curl} \boldsymbol{v}=\frac{\partial v_{y}}{\partial x}-\frac{\partial v_{x}}{\partial y} \in \mathcal{D}^{\prime}(\Omega), \quad \operatorname{div} \boldsymbol{v}=\frac{\partial v_{x}}{\partial x}+\frac{\partial v_{y}}{\partial y} \in \mathcal{D}^{\prime}(\Omega) .
$$

For $v \in L^{2}(\Omega)$,

$$
\operatorname{curl} v=\left(\frac{\partial v}{\partial y},-\frac{\partial v}{\partial x}\right) \in \mathcal{D}^{\prime}(\Omega) \times \mathcal{D}^{\prime}(\Omega) .
$$

Let us introduce the functional spaces

$$
\begin{aligned}
\boldsymbol{H}(\operatorname{curl} ; \Omega) & :=\left\{\boldsymbol{u} \in \boldsymbol{L}^{2}(\Omega) \mid \operatorname{curl} \boldsymbol{u} \in L^{2}(\Omega)\right\}, \\
\boldsymbol{H}(\operatorname{div} ; \xi ; \Omega) & :=\left\{\boldsymbol{u} \in \boldsymbol{L}^{2}(\Omega) \mid \operatorname{div}(\xi \boldsymbol{u}) \in L^{2}(\Omega)\right\},
\end{aligned}
$$

where $\xi$ belongs to $L^{\infty}(\Omega)$. Next, let

$$
\boldsymbol{X}_{N}(\Omega, \varepsilon):=\{\boldsymbol{u} \in \boldsymbol{H}(\operatorname{curl} ; \Omega) \cap \boldsymbol{H}(\operatorname{div} ; \varepsilon ; \Omega) \mid \boldsymbol{u} \cdot \boldsymbol{\tau}=0 \text { on } \partial \Omega\} .
$$

Endowed with the norm $\|\boldsymbol{u}\|_{\boldsymbol{X}_{N}(\Omega)}:=\left(\|\boldsymbol{u}\|_{\boldsymbol{L}^{2}(\Omega)}^{2}+\|\operatorname{curl} \boldsymbol{u}\|_{L^{2}(\Omega)}^{2}+\|\operatorname{div}(\varepsilon \boldsymbol{u})\|_{L^{2}(\Omega)}^{2}\right)^{\frac{1}{2}}$, $\boldsymbol{X}_{N}(\Omega, \varepsilon)$ is a Hilbert space. Following Grisvard [18, 19], for any edge $\partial \Omega_{j}^{k}$, we let $\widetilde{H}^{\frac{1}{2}}\left(\partial \Omega_{j}^{k}\right)$ denote the set of elements of $H^{\frac{1}{2}}\left(\partial \Omega_{j}^{k}\right)$ whose continuation by 0 to $\partial \Omega_{j}$ belongs to $H^{\frac{1}{2}}\left(\partial \Omega_{j}\right)$.

For $u \in L^{2}(\Omega)$ (respectively $\boldsymbol{u} \in \boldsymbol{L}^{2}(\Omega)$ ), we use the notation $u_{j}:=\left.u\right|_{\Omega_{j}}$ (resp. $\boldsymbol{u}_{j}:=\left.\boldsymbol{u}\right|_{\Omega_{j}}$ ). To study the regularity of scalar fields, we consider

$$
P H^{s}(\Omega, \mathcal{P}):=\left\{u \in L^{2}(\Omega) \mid u_{j} \in H^{s}\left(\Omega_{j}\right), j=1 . . N\right\} \text { for } s>0 .
$$


On $P H^{s}(\Omega, \mathcal{P})$, we introduce the broken norm $\|u\|_{P H^{s}(\Omega)}:=\left(\sum_{j=1}^{N}\left\|u_{j}\right\|_{H^{s}\left(\Omega_{j}\right)}^{2}\right)^{\frac{1}{2}}$. We recall that, for $s<1 / 2,\|u\|_{P H^{s}(\Omega)} \simeq\|u\|_{H^{s}(\Omega)}$. The vector valued counterpart is

$$
P \boldsymbol{H}^{s}(\Omega, \mathcal{P}):=P H^{s}(\Omega, \mathcal{P}) \times P H^{s}(\Omega, \mathcal{P}) .
$$

So, we can introduce the subspace of piecewise smooth elements of $\boldsymbol{X}_{N}(\Omega, \varepsilon)$, which writes

$$
\boldsymbol{H}_{N}(\Omega, \varepsilon):=\boldsymbol{X}_{N}(\Omega, \varepsilon) \cap P \boldsymbol{H}^{1}(\Omega, \mathcal{P}) .
$$

Finally, we define families of weighted functional (Sobolev) spaces. For a 2-index $\alpha=\left(\alpha_{1}, \alpha_{2}\right) \in \mathbb{N}^{2}$, we let

$$
|\alpha|:=\alpha_{1}+\alpha_{2} \text { and } \partial_{\boldsymbol{x}}^{\alpha}:=\frac{\partial^{|\alpha|}}{\partial^{\alpha_{1}} x \partial^{\alpha_{2} y}} .
$$

Given $\mathcal{O}$ any open subset of $\mathbb{R}^{2}, m \in \mathbb{N}$ and $\gamma \in \mathbb{R}$, we introduce

$$
V_{\gamma}^{m}(\mathcal{O}):=\left\{v \in L_{l o c}^{2}(\mathcal{O})\left|r^{|\alpha|-m+\gamma} \partial_{\boldsymbol{x}}^{\alpha} v \in L^{2}(\mathcal{O}), \forall \alpha \in \mathbb{N}^{2},\right| \alpha \mid \leq m\right\}
$$

where $r$ is the distance to some given point of $\overline{\mathcal{O}}$, with norm

$$
\|v\|_{V_{\gamma}^{m}(\mathcal{O})}:=\left(\sum_{|\alpha| \leq m}\left\|r^{|\alpha|-m+\gamma} \partial_{\boldsymbol{x}}^{\alpha} v\right\|_{L^{2}(\mathcal{O})}^{2}\right)^{\frac{1}{2}} .
$$

To conclude, $\stackrel{\circ}{V}_{\gamma}^{m}(\mathcal{O})$ is the closure of $\mathcal{D}(\mathcal{O})$ in $V_{\gamma}^{m}(\mathcal{O})$.

3. A continuous splitting of fields of $\boldsymbol{X}_{N}(\Omega, \varepsilon)$. Let us prove a continuous splitting of fields that belong to $\boldsymbol{X}_{N}(\Omega, \varepsilon)$. This kind of result can be traced back to the works of Birman-Solomyak $[5,4,6,7]$. We give here a proof that follows the lines of those of theorems 3.4 and 3.5 of [14]. By assumption, the partition $\mathcal{P}$ of $\Omega$ is labelled so that $\overline{\Omega_{j}}$ and $\overline{\Omega_{j+1}}$ share at least one common edge, for $j=1 . . N-1$ : we call $\left(A_{j}\right)_{j=1 . . N-1}$ these edges.

Proposition 3.1. Let $\boldsymbol{u} \in \boldsymbol{X}_{N}(\Omega, \varepsilon)$. There exist $\boldsymbol{u}_{1} \in \boldsymbol{H}_{N}(\Omega, \varepsilon)$ and $\boldsymbol{u}_{2} \in$ $\boldsymbol{X}_{N}(\Omega, \varepsilon)$ with $\left(\text { curl } \boldsymbol{u}_{2}, 1\right)_{L^{2}\left(\Omega_{j}\right)}=0$ for $j=1 . . N$, such that

$$
\boldsymbol{u}=\boldsymbol{u}_{1}+\boldsymbol{u}_{2}
$$

In addition, one has the stability estimate

$$
\left\|\boldsymbol{u}_{1}\right\|_{P \boldsymbol{H}^{1}(\Omega)}+\left\|\boldsymbol{u}_{2}\right\|_{\boldsymbol{X}_{N}(\Omega)} \leq C\|\boldsymbol{u}\|_{\boldsymbol{X}_{N}(\Omega)}
$$

where the constant $C$ is independent of $\boldsymbol{u}$.

Proof. For any $\boldsymbol{w} \in \boldsymbol{X}_{N}(\Omega, \varepsilon)$, we define $m_{j}(\boldsymbol{w}):=(\operatorname{curl} \boldsymbol{w}, 1)_{L^{2}\left(\Omega_{j}\right)}$, for $j=1 . . N$. To prove our claim, let us build explicitly a family $\left(\boldsymbol{f}_{j}\right)_{j=1 . . N-1}$ of elements of $\boldsymbol{H}_{N}(\Omega, \varepsilon)$, such that

$$
\boldsymbol{u}_{2}=\boldsymbol{u}-\sum_{i=1}^{N-1} c_{i} \boldsymbol{f}_{i} \quad \text { with } c_{i}=\sum_{k=1}^{i} m_{k}(\boldsymbol{u}), i=1 . . N-1
$$


automatically fulfills the conditions $m_{j}\left(\boldsymbol{u}_{2}\right)=0, j=1 . . N$.

For $j=1 . . N-1$, let $\boldsymbol{n}_{j}$ be the unit normal vector to $A_{j}$, going from $\Omega_{j}$ to $\Omega_{j+1}$, and $\boldsymbol{\tau}_{j}$ such that $\left(\boldsymbol{\tau}_{j}, \boldsymbol{n}_{j}\right)$ is a direct, orthonormal basis. Let $M_{j}$ be a given interior point of $A_{j}$, and let $r_{j}$ be the distance to $M_{j}$. Next, define $\boldsymbol{f}_{j}:=C_{j} \zeta_{j}\left(r_{j}\right) \boldsymbol{\tau}_{j}$, with a positive-valued function $\zeta_{j} \in \mathcal{D}\left(\mathbb{R}^{+}\right)$, equal to 1 in a neighborhood of 0 , with support such that supp $\boldsymbol{f}_{j} \cap \mathcal{S}=\emptyset$ and $\left(\operatorname{supp} \boldsymbol{f}_{j} \cap \mathcal{A}\right) \subset A_{j}$. The constant $C_{j}$ is chosen so that $\left(\operatorname{curl} \boldsymbol{f}_{j}, 1\right)_{L^{2}\left(\Omega_{j}\right)}=\int_{A_{j}} \boldsymbol{f}_{j} \cdot \boldsymbol{\tau}_{j}=1$. Note that, by construction, $\boldsymbol{f}_{j}\left|\Omega_{j} \cdot \boldsymbol{\tau}-\boldsymbol{f}_{j}\right|_{\Omega_{j+1}} \cdot \boldsymbol{\tau}=0$ and $\varepsilon \boldsymbol{f}_{j}\left|\Omega_{j} \cdot \boldsymbol{n}_{j}-\varepsilon \boldsymbol{f}_{j}\right|_{\Omega_{j+1}} \cdot \boldsymbol{n}_{j}=0$. Since $\boldsymbol{f}_{j}$ is smooth on $\Omega_{j}$ and $\Omega_{j+1}$ (and supported in $\overline{\Omega_{j}} \cup \overline{\Omega_{j+1}}$ ), we infer that $\boldsymbol{f}_{j} \in \boldsymbol{H}_{N}(\Omega, \varepsilon)$.

Now, let $\boldsymbol{u}_{2}=\boldsymbol{u}-\sum_{i=1}^{N-1} c_{i} \boldsymbol{f}_{i} \in \boldsymbol{X}_{N}(\Omega, \varepsilon)$ be defined as in (3.2), and let us check that $m_{j}\left(\boldsymbol{u}_{2}\right)=0, j=1 . . N$. First, for $1 \leq j \leq N, 1 \leq i \leq N-1$, we remark that

$$
m_{j}\left(\boldsymbol{f}_{i}\right)= \begin{cases}1 & \text { if } j=i \\ -1 & \text { if } j=i+1 \\ 0 & \text { else }\end{cases}
$$

So, we have

$$
m_{1}\left(\boldsymbol{u}_{2}\right)=m_{1}(\boldsymbol{u})-\sum_{i=1}^{N-1} c_{i} m_{1}\left(\boldsymbol{f}_{i}\right)=m_{1}(\boldsymbol{u})-c_{1}=0,
$$

and, for $j=2 . . N-1$,

$$
m_{j}\left(\boldsymbol{u}_{2}\right)=m_{j}(\boldsymbol{u})-\sum_{i=1}^{N-1} c_{i} m_{j}\left(\boldsymbol{f}_{i}\right)=m_{j}(\boldsymbol{u})-c_{j}+c_{j-1}=0 .
$$

On the other hand,

$$
m_{N}\left(\boldsymbol{u}_{2}\right)=m_{N}(\boldsymbol{u})+c_{N-1}=\sum_{i=1}^{N} m_{i}(\boldsymbol{u})=0 \text { because } \boldsymbol{u} \cdot \boldsymbol{\tau}=0 \text { on } \partial \Omega
$$

Finally, let $\boldsymbol{u}_{1}:=\sum_{i=1}^{N-1} c_{i} \boldsymbol{f}_{i} \in \boldsymbol{H}_{N}(\Omega, \varepsilon)$. For $i=1 . . N-1$, there holds $\left|c_{i}\right| \leq$ $\sum_{k=1}^{i}\left|m_{k}(\boldsymbol{u})\right| \leq \sum_{k=1}^{i} \sqrt{\left|\Omega_{k}\right|}\|\operatorname{curl} \boldsymbol{u}\|_{L^{2}\left(\Omega_{k}\right)} \leq \sum_{k=1}^{i} \sqrt{\left|\Omega_{k}\right|}\|\boldsymbol{u}\|_{\boldsymbol{X}_{N}(\Omega)}$. As a consequence, $\left\|\boldsymbol{u}_{1}\right\|_{P \boldsymbol{H}^{1}(\Omega)} \leq C_{1}\|\boldsymbol{u}\|_{\boldsymbol{X}_{N}(\Omega)}$. This leads to (3.1), as

$$
\left\|\boldsymbol{u}_{2}\right\|_{\boldsymbol{X}_{N}(\Omega)} \leq\left\|\boldsymbol{u}_{1}\right\|_{\boldsymbol{X}_{N}(\Omega)}+\|\boldsymbol{u}\|_{\boldsymbol{X}_{N}(\Omega)} \leq C_{2}\left\|\boldsymbol{u}_{1}\right\|_{P_{\boldsymbol{H}^{1}(\Omega)}}+\|\boldsymbol{u}\|_{\boldsymbol{X}_{N}(\Omega)} .
$$

Theorem 3.2. Let $\boldsymbol{u} \in \boldsymbol{X}_{N}(\Omega, \varepsilon)$. There exist $\boldsymbol{u}_{0} \in \boldsymbol{H}_{N}(\Omega, \varepsilon)$ and $\varphi \in H_{0}^{1}(\Omega)$ with $\operatorname{div}(\varepsilon \nabla \varphi) \in L^{2}(\Omega)$, such that

$$
\boldsymbol{u}=\boldsymbol{u}_{0}+\nabla \varphi
$$

Furthermore, one has the stability estimate

$$
\left\|\boldsymbol{u}_{0}\right\|_{P_{\boldsymbol{H}^{1}(\Omega)}}+\|\nabla \varphi\|_{\boldsymbol{L}^{2}(\Omega)}+\|\operatorname{div}(\varepsilon \nabla \varphi)\|_{L^{2}(\Omega)} \leq C\|\boldsymbol{u}\|_{\boldsymbol{X}_{N}(\Omega)}
$$

where the constant $C$ is independent of $u$.

Proof. Let $\boldsymbol{u} \in \boldsymbol{X}_{N}(\Omega, \varepsilon)$. Thanks to proposition 3.1, there exist $\boldsymbol{u}_{1} \in \boldsymbol{H}_{N}(\Omega, \varepsilon)$ and $\boldsymbol{u}_{2} \in \boldsymbol{X}_{N}(\Omega, \varepsilon)$ such that $\boldsymbol{u}=\boldsymbol{u}_{1}+\boldsymbol{u}_{2}$, with $\left(\operatorname{curl} \boldsymbol{u}_{2}, 1\right)_{L^{2}\left(\Omega_{j}\right)}=0$, for $j=1 . . N$. In addition, we have the estimate

$$
\left\|\boldsymbol{u}_{1}\right\|_{P \boldsymbol{H}^{1}(\Omega)}+\left\|\boldsymbol{u}_{2}\right\|_{\boldsymbol{X}_{N}(\Omega)} \leq C_{1}\|\boldsymbol{u}\|_{\boldsymbol{X}_{N}(\Omega)} .
$$


Let us begin by a study of $\boldsymbol{u}_{2}$. Thanks to the assumption $\left(\operatorname{curl} \boldsymbol{u}_{2}, 1\right)_{L^{2}\left(\Omega_{j}\right)}=0$, for every $j=1 . . N$, there exist one, and only one up to a constant, $\phi_{j}$ in $H^{1}\left(\Omega_{j}\right)$ that solves

$$
\mid \begin{array}{lll}
-\Delta \phi_{j}=\operatorname{curl} \operatorname{curl} \phi_{j} & =\operatorname{curl} \boldsymbol{u}_{2} & \text { in } \Omega_{j} \\
\frac{\partial \phi_{j}}{\partial \boldsymbol{n}_{j}} & =0 & \text { on } \partial \Omega_{j} .
\end{array}
$$

All polygons $\Omega_{j}$ are convex, so $\phi_{j} \in H^{2}\left(\Omega_{j}\right)$ (cf. theorem 2.4 .3 of [19]). Next, we define $\boldsymbol{v} \in \boldsymbol{L}^{2}(\Omega)$ by $\left.\boldsymbol{v}\right|_{\Omega_{j}}=\operatorname{curl} \phi_{j}$, for $j=1 . . N$ : it belongs to $P \boldsymbol{H}^{1}(\Omega, \mathcal{P})$, and $\|\boldsymbol{v}\|_{P \boldsymbol{H}^{1}(\Omega)} \leq C_{2}\left\|\operatorname{curl} \boldsymbol{u}_{2}\right\|_{L^{2}(\Omega)}$. In addition, for $j=1 . . N, k=1 . . n_{j}$, there holds

$$
\boldsymbol{v} \cdot \boldsymbol{\tau}_{j}^{k}=\frac{\partial \phi_{j}}{\partial \boldsymbol{n}_{j}^{k}}=0
$$

where $\boldsymbol{\tau}_{j}^{k}$ is such that $\left(\boldsymbol{\tau}_{j}^{k}, \boldsymbol{n}_{j}^{k}\right)$ is a direct, orthonormal basis. Consequently, curl $\boldsymbol{v}$ belongs to $L^{2}(\Omega)$, and $\boldsymbol{w}:=\boldsymbol{u}_{2}-\boldsymbol{v} \in \boldsymbol{H}(\operatorname{curl} ; \Omega)$ is curl-free. According to theorem 2.9 of [17, chapter 1] and thanks to the connectedness of $\partial \Omega$, there exists one, and only one $\varphi_{1} \in H_{0}^{1}(\Omega)$ such that

$$
\boldsymbol{w}=\nabla \varphi_{1}
$$

So far, $\boldsymbol{u}$ has been split as

$$
\boldsymbol{u}=\boldsymbol{u}_{1}+\boldsymbol{v}+\nabla \varphi_{1}
$$

where $\boldsymbol{u}_{1} \in \boldsymbol{H}_{N}(\Omega, \varepsilon), \boldsymbol{v} \in P \boldsymbol{H}^{1}(\Omega)$ and $\varphi_{1} \in H_{0}^{1}(\Omega)$. However, except when $\varepsilon$ is constant over $\Omega, \operatorname{div}(\varepsilon \boldsymbol{v})$ does not belong to $L^{2}(\Omega)$. Using either theorem 1.5.2.8 of [18] or theorem 1 of [3], one can lift the normal trace of $\boldsymbol{v}_{j}$ on $\partial \Omega_{j}$, resulting in $r \in P H^{2}(\Omega, \mathcal{P}) \cap H_{0}^{1}(\Omega)$ such that $r_{j} \in H^{2}\left(\Omega_{j}\right) \cap H_{0}^{1}\left(\Omega_{j}\right)$ that fulfills

$$
\begin{aligned}
& \left.\quad \frac{\partial r_{j}}{\partial \boldsymbol{n}_{j}^{k}}\right|_{\partial \Omega_{j}^{k}}=\left.\left(\boldsymbol{v}_{j} \cdot \boldsymbol{n}_{j}^{k}\right)\right|_{\partial \Omega_{j}^{k}}, k=1 . . n_{j}, j=1 . . N \\
& \text { and }\|r\|_{P H^{2}(\Omega)} \leq C_{3}\|\boldsymbol{v}\|_{P \boldsymbol{H}^{1}(\Omega)} .
\end{aligned}
$$

Note that this is possible as soon as $\left.\left(\boldsymbol{v}_{j} \cdot \boldsymbol{n}_{j}^{k}\right)\right|_{\partial \Omega_{j}^{k}} \in \widetilde{H}^{\frac{1}{2}}\left(\partial \Omega_{j}^{k}\right)$ : as a matter of fact, there holds $\boldsymbol{v}_{j} \cdot \boldsymbol{n}_{j}^{k}=\frac{\partial \phi_{j}}{\partial \boldsymbol{\tau}_{j}^{k}} \in \widetilde{H}^{\frac{1}{2}}\left(\partial \Omega_{j}^{k}\right)$, because $\frac{\partial \phi_{j}}{\partial \boldsymbol{n}_{j}^{k^{\prime}}}=0$ for all $k^{\prime}=1 . . n_{j}$. Define next $\boldsymbol{u}_{3}:=\boldsymbol{v}-\nabla r$. This field is such that

$$
\begin{aligned}
\text { i) } & \boldsymbol{u}_{3} \in \boldsymbol{L}^{2}(\Omega) \\
\text { ii) } & \left.\boldsymbol{u}_{3}\right|_{\Omega_{j}} \in \boldsymbol{H}^{1}\left(\Omega_{j}\right) \\
\text { iii) } & \operatorname{curl} \boldsymbol{u}_{3}=\operatorname{curl} \boldsymbol{v}=\operatorname{curl} \boldsymbol{u}_{2} \in L^{2}(\Omega) \\
\text { iv) } & \left.\boldsymbol{u}_{3} \cdot \boldsymbol{\tau}\right|_{\partial \Omega}=\left.\boldsymbol{v} \cdot \boldsymbol{\tau}\right|_{\partial \Omega}-\left.\nabla r \cdot \boldsymbol{\tau}\right|_{\partial \Omega} \underset{\operatorname{cf.}\left({ }^{3.4)}\right.}{=}-\left.\nabla r \cdot \boldsymbol{\tau}\right|_{\partial \Omega} \underset{r \in H_{0}^{1}(\Omega)}{=} 0 .
\end{aligned}
$$

Now, as $\left.\left(\left.\varepsilon_{j} \boldsymbol{u}_{3}\right|_{\Omega_{j}} \cdot \boldsymbol{n}_{j}^{k}\right)\right|_{\partial \Omega_{j}^{k}}=0, k=1 . . n_{j}, j=1 . . N$, one obtains $\operatorname{div}\left(\varepsilon \boldsymbol{u}_{3}\right) \in L^{2}(\Omega)$. Combining those arguments allows us to conclude that $\boldsymbol{u}_{3} \in \boldsymbol{H}_{N}(\Omega, \varepsilon)=\boldsymbol{X}_{N}(\Omega, \varepsilon) \cap$ $P \boldsymbol{H}^{1}(\Omega, \mathcal{P})$.

Let $\varphi:=\varphi_{1}+r \in H_{0}^{1}(\Omega)$ and $\boldsymbol{u}_{0}:=\boldsymbol{u}_{1}+\boldsymbol{u}_{3}$. By construction

$$
\boldsymbol{u}=\boldsymbol{u}_{0}+\nabla \varphi
$$


with $\operatorname{div}(\varepsilon \nabla \varphi)=\operatorname{div}(\varepsilon \boldsymbol{u})-\operatorname{div}\left(\varepsilon \boldsymbol{u}_{0}\right) \in L^{2}(\Omega)$. Also

$$
\begin{aligned}
\left\|\boldsymbol{u}_{0}\right\|_{P \boldsymbol{H}^{1}(\Omega)} & \leq\left\|\boldsymbol{u}_{1}\right\|_{P \boldsymbol{H}^{1}(\Omega)}+\|\boldsymbol{v}\|_{P \boldsymbol{H}^{1}(\Omega)}+\|\nabla r\|_{P \boldsymbol{H}^{1}(\Omega)} \\
& \leq\left\|\boldsymbol{u}_{1}\right\|_{P \boldsymbol{H}^{1}(\Omega)}+\left(1+C_{3}\right)\|\boldsymbol{v}\|_{P \boldsymbol{H}^{1}(\Omega)} \\
& \leq\left\|\boldsymbol{u}_{1}\right\|_{P_{\boldsymbol{H}^{1}}(\Omega)}+C_{2}\left(1+C_{3}\right)\left\|\operatorname{curl} \boldsymbol{u}_{2}\right\|_{L^{2}(\Omega)} \\
& \leq C_{1}\left(1+C_{2}\left(1+C_{3}\right)\right)\|\boldsymbol{u}\|_{\boldsymbol{X}_{N}(\Omega)} \\
\|\nabla \varphi\|_{\boldsymbol{L}^{2}(\Omega)} & \leq\left\|\boldsymbol{u}-\boldsymbol{u}_{0}\right\|_{\boldsymbol{L}^{2}(\Omega)} \\
& \leq\|\boldsymbol{u}\|_{\boldsymbol{X}_{N}(\Omega)}+\left\|\boldsymbol{u}_{0}\right\|_{\boldsymbol{L}^{2}(\Omega)} \\
& \leq\left(1+C_{4}\right)\|\boldsymbol{u}\|_{\boldsymbol{X}_{N}(\Omega)}
\end{aligned}
$$

and

$$
\begin{aligned}
\|\operatorname{div}(\varepsilon \nabla \varphi)\|_{L^{2}(\Omega)} & \leq\|\operatorname{div}(\varepsilon \boldsymbol{u})\|_{L^{2}(\Omega)}+\left\|\operatorname{div}\left(\varepsilon \boldsymbol{u}_{0}\right)\right\|_{L^{2}(\Omega)} \\
& \leq\|\operatorname{div}(\varepsilon \boldsymbol{u})\|_{L^{2}(\Omega)}+C_{5}\left\|\boldsymbol{u}_{0}\right\|_{P_{\boldsymbol{H}^{1}(\Omega)}} \\
& \leq\left(1+C_{4} C_{5}\right)\|\boldsymbol{u}\|_{\boldsymbol{X}_{N}(\Omega)}
\end{aligned}
$$

where $C_{4}:=C_{1}\left(1+C_{2}\left(1+C_{3}\right)\right)$ et $C_{5}:=\max _{j}\left|\varepsilon_{j}\right|$.

4. A study of the regularity. Recall that $\Omega$ is a bounded, open, connected polygonal set of $\mathbb{R}^{2}$, with connected boundary $\partial \Omega$. In this section, we show that, under some assumptions that will be described later on, the scalar potential $\varphi \in H_{0}^{1}(\Omega)$ that appears in the splitting of theorem 3.2 is actually "more regular than $H^{1}$ ". More precisely, we prove that there exists $\sigma_{0}>1$, which depends only on $\Omega$, on the partition and on $\varepsilon$, such that one has $\varphi \in \cap_{s<\sigma_{0}} H^{s}(\Omega)$.

Let us consider the unbounded operator $F$ in $L^{2}(\Omega)$ :

$$
\left\{\begin{array}{ll}
D(F) & :=\left\{u \in H_{0}^{1}(\Omega) \mid \operatorname{div}(\varepsilon \nabla u) \in L^{2}(\Omega)\right\} \\
F u & :=\operatorname{div}(\varepsilon \nabla u)
\end{array} .\right.
$$

Now, we study the regularity of an element $u \in D(F)$. From time to time, we shall use the notation $f:=F u=\operatorname{div}(\varepsilon \nabla u) \in L^{2}(\Omega)$.

Let us begin by some well-known results. Classically (chapter 2, volume 1 of [22]; theorem 2.1.3 of [19], or [2]), the following interior regularity holds.

THEOREM 4.1. Let $\mathcal{O}$ be an open subset of $\Omega$ such that $\overline{\mathcal{O}} \cap \mathcal{S}=\overline{\mathcal{O}} \cap \mathcal{A}=\emptyset$. Then $u$ belongs to $H^{2}(\mathcal{O})$, with estimate

$$
\|u\|_{H^{2}(\mathcal{O})} \leq C\left(\|\operatorname{div}(\varepsilon \nabla u)\|_{L^{2}(\Omega)}+\|\nabla u\|_{L^{2}(\Omega)}\right)
$$

where the constant $C$ is independent of $u$.

Furthermore, theorem 2.1.4 of [19] provides the following regularity result around exterior edges.

THEOREM 4.2. Let $\mathcal{O}$ be an open subset of $\Omega$ such that $\overline{\mathcal{O}} \cap \mathcal{S}=\overline{\mathcal{O}} \cap \mathcal{A}_{\text {int }}=\emptyset$. Then $u$ belongs to $H^{2}(\mathcal{O})$, with estimate

$$
\|u\|_{H^{2}(\mathcal{O})} \leq C\left(\|\operatorname{div}(\varepsilon \nabla u)\|_{L^{2}(\Omega)}+\|\nabla u\|_{L^{2}(\Omega)}\right)
$$

where the constant $C$ is independent of $u$.

4.1. Regularity at interior edges: preliminaries. Let $M$ be an interior point of $A \in \mathcal{A}_{\text {int }}$. Assume that $\Omega_{1}$ and $\Omega_{2}$ are the two subdomains of $\mathcal{P}$ such that $\overline{\Omega_{1}} \cap \overline{\Omega_{2}}=A$. Consider $d$ small enough so that $\overline{B(M, d)} \cap \mathcal{S}=\emptyset$ and $(\overline{B(M, d)} \cap \mathcal{A}) \subset A$ 
where $B(M, d)$ denotes the open ball of centre $M$ and radius $d$. Evidently, the fact that the value of $\varepsilon$ jumps at $A$ prevents a priori $u$ to belong to $H^{2}(B(M, d))$. However, using a technique based on partial Fourier transform alongside one direction, we shall prove that $u_{j} \in H^{2}\left(B(M, d) \cap \Omega_{j}\right), j=1,2$. The proof of this result can be found in [16] (theorem 2.1). We will give a slightly different version using the $T$-coercivity approach (see $[10,8]$ for more details concerning this technique).

Let us begin by some geometric notations. Let $(r, \theta)$ denote the polar coordinates with respect to $M$. The angular coordinate $\theta$ is chosen arbitrarily. Let $\chi \in \mathcal{D}\left(\mathbb{R}^{+}\right)$ be such that $0 \leq \chi \leq 1$, equal to 1 on $[0 ; d]$, with support contained in $\left[0 ; d_{M}\right]$. Here $d_{M}>d$ is small enough so that $\overline{B\left(M, d_{M}\right)} \cap \mathcal{S}=\emptyset$ and $\left(\overline{B\left(M, d_{M}\right)} \cap \mathcal{A}\right) \subset A$. Define the radial cutoff function $\chi_{M}:(r, \theta) \mapsto \chi(r)$ and the infinite strips $\mathcal{I}:=\mathbb{R} \times I, \mathcal{I}_{j}:=\mathbb{R} \times I_{j}$, $j=1,2$, with respectively $I:=]-d_{M} ; d_{M}\left[, I_{1}:=\right]-d_{M} ; 0\left[\right.$ and $\left.I_{2}:=\right] 0 ; d_{M}[$. Without loss of generality, we suppose that $A \subset \mathbb{R} \times\{0\},\left(B\left(M, d_{M}\right) \cap \Omega_{j}\right) \subset \mathcal{I}_{j}, j=1,2$.

Now, we localize the study of the regularity with the help of $\chi_{M}$. Let $\tilde{f}$ be the extension of $f=F u=\operatorname{div}(\varepsilon \nabla u)$ by 0 to $\mathcal{I}$. Consider $\underline{u}:=\chi_{M} u$; $\underline{u}$ belongs to $H_{0}^{1}\left(B\left(M, d_{M}\right)\right)$, so its extension $w:=\underline{\tilde{u}}$ by 0 to $\mathcal{I}$ belongs to $H^{1}(\mathcal{I})$. Consider next

$$
p:=\varepsilon\left(\underline{\tilde{u}} \Delta \chi_{M}+2 \nabla \underline{\tilde{u}} \cdot \nabla \chi_{M}\right)+\tilde{f} \chi_{M},
$$

which belongs to $L^{2}(\mathcal{I})$, with compact support. According to its definition, $w$ is a solution to the transmission problem in the infinite strip $\mathcal{I}$

$$
\left(\mathcal{P}_{\text {strip }}\right) \mid \begin{array}{ll}
\text { Find } w \in H^{1}(\mathcal{I}) & \text { such that } \\
\varepsilon_{j} \Delta w_{j}=p_{j} & \text { in } \mathcal{I}_{j}, j=1,2 \\
w_{j}=0 & \text { on } \partial \mathcal{I}_{j} \cap \partial \mathcal{I}, j=1,2 \\
w_{1}-w_{2}=0 & \text { on } \partial \mathcal{I}_{1} \cap \partial \mathcal{I}_{2} \\
\varepsilon_{1} \partial_{y} w_{1}-\varepsilon_{2} \partial_{y} w_{2}=0 & \text { on } \partial \mathcal{I}_{1} \cap \partial \mathcal{I}_{2} .
\end{array}
$$

4.2. Regularity at interior edges: Fourier transform. Applying the Fourier transform with respect to $x$ to the equations of $\left(\mathcal{P}_{\text {strip }}\right)$ for $\lambda \in \mathbb{R} i$, one finds that $y \mapsto \hat{w}(\lambda, y):=\int_{-\infty}^{+\infty} e^{-\lambda x} w(x, y) d x$ is a solution to

$$
\begin{array}{c|l}
\left(\hat{\mathcal{P}}_{\text {strip }}\right) & \begin{array}{l}
\varepsilon_{j}\left(\partial_{y}^{2}+\lambda^{2}\right) \hat{w}_{j}(\lambda, y)=\hat{p}_{j}(\lambda, y) \quad \text { in } \mathcal{I}_{j}, j=1,2 \\
\hat{w}_{1}\left(\lambda,-d_{M}\right)=\hat{w}_{2}\left(\lambda, d_{M}\right)=0 \\
\hat{w}_{1}(\lambda, 0)=\hat{w}_{2}(\lambda, 0) \\
\varepsilon_{1} \partial_{y} \hat{w}_{1}(\lambda, 0)=\varepsilon_{2} \partial_{y} \hat{w}_{2}(\lambda, 0) .
\end{array}
\end{array}
$$

Let us introduce the symbol related to $\left(\hat{\mathcal{P}}_{\text {strip }}\right)$

$$
\begin{aligned}
& \mathcal{L}_{\text {strip }}(\lambda): \quad D\left(\mathcal{L}_{\text {strip }}\right) \quad \longrightarrow \quad L^{2}(I) \\
& v \quad \longmapsto\left(\varepsilon \lambda^{2}+d_{y} \varepsilon d_{y}\right) v
\end{aligned}
$$

where $D\left(\mathcal{L}_{\text {strip }}\right):=\left\{v \in H_{0}^{1}(I) \mid v_{j} \in H^{2}\left(I_{j}\right), j=1,2, \varepsilon_{1} v_{1}^{\prime}(0)=\varepsilon_{2} v_{2}^{\prime}(0)\right\}$.

LemMA 4.3. Assume that $\varepsilon_{1}+\varepsilon_{2} \neq 0$. Then $\mathcal{L}_{\text {strip }}(\lambda)$ is an isomorphism from $D\left(\mathcal{L}_{\text {strip }}\right)$ to $L^{2}(I)$ for all $\lambda \in \mathbb{R} i$.

Proof. Denote respectively $(\cdot, \cdot),(\cdot, \cdot)_{1},(\cdot, \cdot)_{2}$ the scalar products of $L^{2}(I), L^{2}\left(I_{1}\right)$ and $L^{2}\left(I_{2}\right)$. Define $\tau:=i \lambda \in \mathbb{R}$. If $\psi$ is a measurable function on $I$, we will denote $\psi_{j}:=\left.\psi\right|_{I_{j}}, j=1,2$. Let us introduce the sesquilinear form associated with $\mathcal{L}_{\text {strip }}(\lambda)$. For $v, \psi \in H_{0}^{1}(I)$, it is defined by

$$
a(v, \psi):=\sum_{j=1}^{2}\left(\varepsilon_{j}\left(v_{j}^{\prime}, \psi_{j}^{\prime}\right)_{j}+\tau^{2} \varepsilon_{j}\left(v_{j}, \psi_{j}\right)_{j}\right) .
$$


Let us distinguish two cases according to the value of the ratio $\varepsilon_{2} / \varepsilon_{1}$.

- If $\varepsilon_{2} / \varepsilon_{1} \in \mathbb{C} \backslash \mathbb{R}^{-}$, then the form $a$ is coercive on $H_{0}^{1}(I)$.

Thus, for every $g \in H^{-1}(I)$, there exists a unique $v \in H_{0}^{1}(I)$ such that $\left(\varepsilon \lambda^{2}+\right.$ $\left.d_{y} \varepsilon d_{y}\right) v=g$ in $I$. If moreover $g$ belongs to $L^{2}(I)$ then $d_{y}^{2} v_{1} \in L^{2}\left(I_{1}\right)$ so $v_{1} \in H^{2}\left(I_{1}\right)$. Similarly, $v_{2} \in H^{2}\left(I_{2}\right)$. Since $\mathcal{L}_{\text {sym }}(\lambda)$ is continuous from $D\left(\mathcal{L}_{\text {strip }}\right)$ to $L^{2}(I)$, one can use the open mapping theorem to conclude that $\mathcal{L}_{\text {strip }}$ is an isomorphism for all $\lambda \in \mathbb{R} i$.

- If $\varepsilon_{2} / \varepsilon_{1} \in \mathbb{R}_{*}^{-}$, the form $a$ is no more coercive on $H_{0}^{1}(I)$. Note that, as $\varepsilon_{1}+\varepsilon_{2} \neq 0$, we know that $\varepsilon_{2} / \varepsilon_{1} \neq-1$.

To address this difficulty, we use the $T$-coercivity method, cf. [10, 8]. Introduce the operators $R_{1}, R_{2}$ such that $\left(R_{1} v_{1}\right)(y)=v_{1}(-y),\left(R_{2} v_{2}\right)(y)=v_{2}(-y)$ and the isomorphisms $\left(T_{1} \circ T_{1}=T_{2} \circ T_{2}=I d\right)$ of $H_{0}^{1}(I)$ respectively defined by

$$
T_{1} v:=\left\{\begin{array}{ll}
v_{1} & \text { on } I_{1} \\
-v_{2}+2 R_{1} v_{1} & \text { on } I_{2}
\end{array} \text { and } T_{2} v:=\left\{\begin{array}{ll}
v_{1}-2 R_{2} v_{2} & \text { on } I_{1} \\
-v_{2} & \text { on } I_{2}
\end{array} .\right.\right.
$$

For all $v \in H_{0}^{1}(I)$, one can write, using Young's inequality, for all $\eta>0$,

$$
\begin{aligned}
\left|\varepsilon_{1}^{-1} a\left(v, T_{1} v\right)\right|= & \left|\left(v_{1}^{\prime}, v_{1}^{\prime}\right)_{1}+\tau^{2}\left(v_{1}, v_{1}\right)_{1}+\right| \varepsilon_{2} / \varepsilon_{1} \mid\left(\left(v_{2}^{\prime}, v_{2}^{\prime}\right)_{2}+\tau^{2}\left(v_{2}, v_{2}\right)_{2}\right) \\
& +2\left(\varepsilon_{2} / \varepsilon_{1}\right)\left(\left(v_{2}^{\prime},\left(R_{1} v_{1}\right)^{\prime}\right)_{2}+\tau^{2}\left(v_{2},\left(R_{1} v_{1}\right)\right)_{2}\right) \mid \\
\geq & \left(1-\eta^{-1}\left|\varepsilon_{2} / \varepsilon_{1}\right|\right)\left(\left(v_{1}^{\prime}, v_{1}^{\prime}\right)_{1}+\tau^{2}\left(v_{1}^{\prime}, v_{1}^{\prime}\right)_{1}\right) \\
& +\left|\varepsilon_{2} / \varepsilon_{1}\right|(1-\eta)\left(\left(v_{2}^{\prime}, v_{2}^{\prime}\right)_{2}+\tau^{2}\left(v_{2}^{\prime}, v_{2}^{\prime}\right)_{2}\right) .
\end{aligned}
$$

Thus, if $\left|\varepsilon_{2} / \varepsilon_{1}\right|<1$, taking $\eta$ such that $\left|\varepsilon_{2} / \varepsilon_{1}\right|<\eta<1$, one infers the existence of a constant $C$ independent of $\tau$ such that

$$
\left|a\left(v, T_{1} v\right)\right| \geq C\left(\left(v^{\prime}, v^{\prime}\right)+\tau^{2}(v, v)\right), \forall v \in H_{0}^{1}(I) .
$$

Since $T_{1}$ is an isomorphism of $H_{0}^{1}(I)$, this proves that for every $g \in H^{-1}(I)$, there exists a unique $v \in H_{0}^{1}(I)$ such that $\left(\varepsilon \lambda^{2}+d_{y} \varepsilon d_{y}\right) v=g$.

One proceeds similarly in the case $\left|\varepsilon_{2} / \varepsilon_{1}\right|>1$, working with $T_{2}$.

Finally, we conclude as in the case $\varepsilon_{2} / \varepsilon_{1} \in \mathbb{C} \backslash \mathbb{R}^{-}$.

REMARK 4.4. Notice that the T-coercivity method can not be used to deal with the case $\varepsilon_{2}+\varepsilon_{1}=0$, for which $\varepsilon_{2} / \varepsilon_{1}=-1$. Now, let us study the norm of $\mathcal{L}_{\text {sym }}(\lambda)^{-1}$, which is an operator from $L^{2}(I)$ to $D\left(\mathcal{L}_{\text {sym }}\right)$.

Lemma 4.5. Assume that $\varepsilon_{1}+\varepsilon_{2} \neq 0$. Then there exists a constant $C$ independent of $\lambda \in \mathbb{R} i$ such that

$$
\sum_{j=1}^{2}\left\|v_{j}\right\|_{H^{2}\left(I_{j}\right)}+|\lambda|^{2}\|v\|_{L^{2}(I)} \leq C\left\|\mathcal{L}_{\text {strip }}(\lambda) v\right\|_{L^{2}(I)},
$$

for all $v \in D\left(\mathcal{L}_{\text {strip }}\right)$.

Proof. We prove this result in the case $\varepsilon_{2} / \varepsilon_{1} \in \mathbb{R}_{*}^{-} \backslash\{-1\}$, the case $\varepsilon_{2} / \varepsilon_{1} \in \mathbb{C} \backslash \mathbb{R}^{-}$ being easier to tackle. Consider $v \in D\left(\mathcal{L}_{\text {sym }}\right)$ and denote $g=\mathcal{L}_{\text {sym }}(\lambda) v$. Suppose $\left|\varepsilon_{2} / \varepsilon_{1}\right|<1$. According to (4.3), one can write $C|\lambda|^{2}(v, v) \leq a\left(v, T_{1} v\right)=-\left(g, T_{1} v\right)$, with $C>0$ independent of $\lambda$. Noticing that $T_{1}$ is a continuous operator from $L^{2}(I)$ to $L^{2}(I)$, one obtains the estimation

$$
|\lambda|^{2}\|v\|_{L^{2}(I)} \leq C\|g\|_{L^{2}(I)}
$$

where $C$ does not depend on $\lambda$. On the other hand, since $\varepsilon_{j}\left(\lambda^{2}+d_{y}^{2}\right) v_{j}=g_{j}, j=1,2$, one has

$$
\left\|d_{y}^{2} v_{j}\right\|_{L^{2}\left(I_{j}\right)} \leq C\|g\|_{L^{2}(I)}, j=1,2
$$


From (4.3), one can also write $C\left(v^{\prime}, v^{\prime}\right) \leq a\left(v, T_{1} v\right)$, hence

$$
\left\|d_{y} v\right\|_{L^{2}(I)} \leq C\|g\|_{L^{2}(I)} .
$$

Using (4.4), (4.5) and (4.6), we can finally assert the existence of $C>0$ independent of $\lambda \in \mathbb{R} i$ such that

$$
\sum_{j=1}^{2}\left\|v_{j}\right\|_{H^{2}\left(I_{j}\right)}+|\lambda|^{2}\|v\|_{L^{2}(I)} \leq C\|g\|_{L^{2}(I)},
$$

for all $v \in D\left(\mathcal{L}_{\text {sym }}\right)$.

Working with $T_{2}$, the case $\left|\varepsilon_{2} / \varepsilon_{1}\right|>1$ can be handled similarly.

Since $\mathcal{L}_{\text {strip }}(\lambda) \hat{w}(\lambda, \cdot)=p(\lambda, \cdot)$, thanks to lemma 4.5 , one obtains

$$
\sum_{j=1}^{2}\left\|\hat{w}_{j}(\lambda, \cdot)\right\|_{H^{2}\left(I_{j}\right)}+|\lambda|^{2}\|\hat{w}(\lambda, \cdot)\|_{L^{2}(I)} \leq C\|\hat{p}(\lambda, \cdot)\|_{L^{2}(I)}, \quad \forall \lambda \in \mathbb{R} i .
$$

Above, $C>0$ is independent of $\lambda$.

With the help of the Parseval identity (see the lemma 5.2.4 of [21]), one deduces that $w_{j} \in H^{2}\left(\mathcal{I}_{j}\right), j=1,2$. Besides, there holds the estimate

$$
\left\|w_{1}\right\|_{H^{2}\left(\mathcal{I}_{1}\right)}+\left\|w_{2}\right\|_{H^{2}\left(\mathcal{I}_{2}\right)} \leq C\|p\|_{L^{2}(\mathcal{I})} .
$$

Using the expression (4.2) of $p$ and noticing that $\chi_{M}=1$ over $B(M, d)$, one concludes

$$
\sum_{j=1}^{2}\left\|u_{j}\right\|_{H^{2}\left(B(M, d) \cap \Omega_{j}\right)} \leq C\left(\|\operatorname{div}(\varepsilon \nabla u)\|_{L^{2}(\Omega)}+\|\nabla u\|_{L^{2}(\Omega)}\right) .
$$

Let us summarize this result with the

THEOREM 4.6. Let $\mathcal{O}$ be an open subset of $\Omega$ such that $\overline{\mathcal{O}} \cap \mathcal{S}=\emptyset$ and $(\overline{\mathcal{O}} \cap \mathcal{A}) \subset$ $A=\overline{\Omega_{i}} \cap \overline{\Omega_{j}}$. Under the assumption $\varepsilon_{i}+\varepsilon_{j} \neq 0, u_{i}$ belongs to $H^{2}\left(\mathcal{O} \cap \Omega_{i}\right), u_{j}$ belongs to $H^{2}\left(\mathcal{O} \cap \Omega_{j}\right)$, with estimate

$$
\left\|u_{i}\right\|_{H^{2}\left(\mathcal{O} \cap \Omega_{i}\right)}+\left\|u_{j}\right\|_{H^{2}\left(\mathcal{O} \cap \Omega_{j}\right)} \leq C\left(\|\operatorname{div}(\varepsilon \nabla u)\|_{L^{2}(\Omega)}+\|\nabla u\|_{L^{2}(\Omega)}\right)
$$

where the constant $C$ is independent of $u$.

4.3. Regularity at boundary vertices: preliminaries. To carry out the study in the neighborhood of boundary vertices, we shall follow the method of proof given in [16], which relies itself on the founding paper of Kondrat'ev [20].

As in the study of the regularity at interior edges, let us begin by some geometric notations. For $S \in \mathcal{S}_{\text {ext }}$, let $(r, \theta)$ denote the polar coordinates with respect to $S$. The angular coordinate $\theta$ is chosen in such a way that, for $d>0$ small enough, there holds

$$
\overline{B(S, d)} \cap \bar{\Omega}=\left\{(r \cos \theta, r \sin \theta) \mid(r, \theta) \in[0 ; d] \times\left[0 ; \theta_{\max }\right]\right\} .
$$

One has always $\theta_{\max } \leq 2 \pi$.

Next, let $d_{S}>0$ be small enough, so that $\overline{B\left(S, d_{S}\right)} \cap \mathcal{S}=\{S\}$. Let $\chi \in \mathcal{D}\left(\mathbb{R}^{+}\right)$be such that $0 \leq \chi \leq 1$, equal to 1 on $\left[0 ; \frac{d_{S}}{2}\right]$, with support contained in $\left[0 ; d_{S}\right]$. Then, the radial cutoff function $\chi_{S}:(r, \theta) \mapsto \chi(r)$ is such that supp $\chi_{S} \cap \mathcal{S}=\{S\}$, where supp $\chi_{S}$ is the support of $\chi_{S}$. 
Define $\tilde{\Omega}:=\Omega \cap B\left(S, d_{S}\right)$. We number again the $J_{S}$ subdomains that have $S$ as one of their boundary vertices, from 1 to $J_{S}$. Further, if we denote by $\tilde{\Omega}_{j}:=$ $\Omega_{j} \cap B\left(S, d_{S}\right), j=1 \ldots J_{S}$, the new numbering is such that $\bar{\Omega}_{j}$ and $\bar{\Omega}_{j+1}$ share one edge, for $j=1$.. $\left(J_{S}-1\right)$, whose angle $\theta$ grows with $j$. Then, for $j=1 . . J_{S}, \delta \sigma_{j}$ denotes the interior opening of $\tilde{\Omega}_{j}$ (with $\delta \sigma_{j} \leq \pi$ because $\tilde{\Omega}_{j}$ is convex), and we set $\sigma_{0}:=0$, and $\sigma_{j}:=\sigma_{j-1}+\delta \sigma_{j}, j=1 . . J_{S}$. By definition, there holds $\sum_{j=1 . .} J_{S} \delta \sigma_{j}=\theta_{\max }$. Finally, we define the intervals $\left.G_{j}:=\right] \sigma_{j-1} ; \sigma_{j}\left[, j=1 . . J_{S}\right.$, and $\left.G:=\right] 0 ; \sigma_{J_{S}}[$, and the unbounded angular sectors

$$
\begin{aligned}
& \Gamma \quad:=\left\{(x, y) \in \mathbb{R}^{2} \mid r>0, \theta \in G\right\}, \\
& \Gamma_{j}:=\left\{(x, y) \in \mathbb{R}^{2} \mid r>0, \theta \in G_{j}\right\}, j=1 . . J_{S} .
\end{aligned}
$$

For $v \in L^{2}(\tilde{\Omega})\left(\operatorname{resp} . v \in L^{2}(\Gamma)\right)$, we write $v_{j}:=\left.v\right|_{\tilde{\Omega}_{j}}\left(\right.$ resp. $\left.v_{j}:=\left.v\right|_{\Gamma_{j}}\right), j=1 \ldots J_{S}$.

We introduce $\Lambda_{\varepsilon, S}$, the set of singular exponents related to the vertex $S$, which we define as the set of complex numbers $\lambda \in \mathbb{C}$ such that there is a non-zero $J_{S}$-tuple $\left(\phi_{\lambda, j}\right)_{j=1}^{J_{S}} \in \prod_{j=1}^{J_{S}} H^{2}\left(G_{j}\right)$ which fulfills the conditions below:

$$
\mid \begin{array}{ll}
\left(\partial_{\theta}^{2}+\lambda^{2}\right) \phi_{\lambda, j}=0 & \text { in } G_{j}, j=1 . . J_{S} \\
\phi_{\lambda, 1}(0)=\phi_{\lambda, J_{S}}\left(\sigma_{J_{S}}\right)=0 & \\
\phi_{\lambda, j}\left(\sigma_{j}\right)=\phi_{\lambda, j+1}\left(\sigma_{j}\right) & j=1 . .\left(J_{S}-1\right) \\
\varepsilon_{j} \partial_{\theta} \phi_{\lambda, j}\left(\sigma_{j}\right)=\varepsilon_{j+1} \partial_{\theta} \phi_{\lambda, j+1}\left(\sigma_{j}\right) & j=1 . .\left(J_{S}-1\right) .
\end{array}
$$

Next, we localize the study of the regularity, using $\chi_{S}$. Let $\tilde{f}$ be the extension of $f$ by 0 to $\Gamma$. Next consider $\underline{u}:=\chi_{S} u$ : $\underline{u}$ belongs to $H_{0}^{1}(\tilde{\Omega})$, so its extension $w:=\underline{\tilde{u}}$ by 0 to $\Gamma$ belongs to $H^{1}(\Gamma)$. Consider next

$$
p:=\varepsilon\left(\underline{\tilde{u}} \Delta \chi_{S}+2 \nabla \underline{\tilde{u}} \cdot \nabla \chi_{S}\right)+\tilde{f} \chi_{S},
$$

which belongs to $L^{2}(\Gamma)$, with compact support. According to its definition, $w$ is a solution to the transmission problem

$$
\begin{array}{l|ll}
\left(\mathcal{P}_{\text {sector }}\right) & \text { Find } w \in H^{1}(\Gamma) \text { such that } & \\
\varepsilon_{j} \Delta w_{j}=p_{j} & \text { in } \Gamma_{j}, j=1 . . J_{S} \\
w_{1}=0 & \text { on } \partial \Gamma_{1} \cap \partial \Gamma \\
w_{J_{S}}=0 & \text { on } \partial \Gamma_{J_{S}} \cap \partial \Gamma \\
w_{j}-w_{j+1}=0 & \text { on } \partial \Gamma_{j} \cap \partial \Gamma_{j+1}, j=1 . .\left(J_{S}-1\right) \\
\varepsilon_{j} \partial_{\theta} w_{j}-\varepsilon_{j+1} \partial_{\theta} w_{j+1}=0 & \text { on } \partial \Gamma_{j} \cap \partial \Gamma_{j+1}, j=1 . .\left(J_{S}-1\right) .
\end{array}
$$

4.4. Regularity at boundary vertices: Mellin transform. For the definition of the Mellin transform, let us recall a classical lemma (see §2, chapter 2 of [24], or Annex AA of [15]).

Lemma 4.7. Let $\gamma \in \mathbb{R}$ and $s \in \mathbb{N}$.

If $v \in V_{\gamma}^{s}(\Gamma)$, then one can define its Mellin transform

$$
\hat{v}(\lambda, \cdot):=\mathcal{M} v(\lambda, \cdot)=\int_{0}^{+\infty} r^{-\lambda} v(r, \cdot) \frac{d r}{r}
$$

for $\lambda \in \mathbb{C}$ such that $\Re(\lambda)=s-\gamma-1$. Moreover, one has $\eta \mapsto \hat{v}(\xi+i \eta, \cdot) \in$ $L^{2}\left(\mathbb{R}, H^{s}(G)\right)$, where $\xi:=s-\gamma-1$. 
In the problem of interest, $w$ belongs to $H^{1}(\Gamma)$, with a compact support. So, it also belongs to $V_{\gamma}^{1}(\Gamma)$, for all $\gamma>0$. As a consequence, one can define its Mellin transform $\hat{w}(\lambda, \cdot)$ on the complex lines $\{\lambda \in \mathbb{C} \mid \Re(\lambda)=-\gamma\}$, for all $\gamma>0$. It follows that $\hat{w}(\lambda, \cdot)$ is well-defined over the complex half-plane $\{\lambda \in \mathbb{C} \mid \Re(\lambda)<0\}$.

Next, we shall use $h:=r^{2} p$, where $p$ is defined in (4.8). Since $p \in L^{2}(\Gamma)$ and moreover $p$ is compactly supported, $h$ belongs to $V_{-2+\gamma}^{0}(\Gamma)$, for all $\gamma \geq 0$. In this case, its Mellin transform $\theta \mapsto \hat{h}(\lambda, \theta)$ is well-defined over the complex half-plane $\{\lambda \in \mathbb{C} \mid \Re(\lambda)<1\}$.

Multiplying by $r^{2}$ the volume PDEs in $\left(\mathcal{P}_{\text {sector }}\right)$, and then carrying out the Mellin transform for $\lambda \in \mathbb{C}$ such that $\Re(\lambda)<0$, one finds that $\theta \mapsto \hat{w}(\lambda, \theta)$ is a solution to

$$
\left(\hat{\mathcal{P}}_{\text {sector }}\right) \mid \begin{array}{ll}
\varepsilon_{j}\left(\partial_{\theta}^{2}+\lambda^{2}\right) \hat{w}_{j}(\lambda, \theta)=\hat{h}_{j}(\lambda, \theta) & \text { in } G_{j}, j=1 . . J_{S} \\
\hat{w}_{1}(\lambda, 0)=\hat{w}_{J_{S}}\left(\lambda, \sigma_{J_{S}}\right)=0 & \\
\hat{w}_{j}\left(\lambda, \sigma_{j}\right)=\hat{w}_{j+1}\left(\lambda, \sigma_{j}\right) & j=1 . .\left(J_{S}-1\right) \\
\varepsilon_{j} \partial_{\theta} \hat{w}_{j}\left(\lambda, \sigma_{j}\right)=\varepsilon_{j+1} \partial_{\theta} \hat{w}_{j+1}\left(\lambda, \sigma_{j}\right) & j=1 . .\left(J_{S}-1\right) .
\end{array}
$$

Next, consider the Mellin symbol related to $\left(\hat{\mathcal{P}}_{\text {sector }}\right)$

$$
\begin{aligned}
& \mathcal{L}(\lambda): \quad D(\mathcal{L}) \quad \longrightarrow \quad \prod_{j=1}^{J_{S}} L^{2}\left(G_{j}\right) \\
& v \quad \longmapsto\left(\varepsilon_{j}\left(d_{\theta}^{2}+\lambda^{2}\right) v_{j}\right)_{j=1}^{J_{S}}
\end{aligned}
$$

where $D(\mathcal{L}):=\left\{v \in H_{0}^{1}(G) \mid v_{j} \in H^{2}\left(G_{j}\right), \varepsilon_{j} v_{j}^{\prime}\left(\sigma_{j}\right)=\varepsilon_{j+1} v_{j+1}^{\prime}\left(\sigma_{j}\right), j=1 . .\left(J_{S}-1\right)\right\}$.

Lemma 4.8. Let $\lambda \in \mathcal{B}(-1 ; 1)$. Then, $\mathcal{L}(\lambda)$ is a bijective map from $D(\mathcal{L})$ to $\prod_{j=1}^{J_{S}} L^{2}\left(G_{j}\right)$ if, and only if, there holds $\lambda \notin \Lambda_{\varepsilon, S}$.

Proof. • If $\lambda \in \Lambda_{\varepsilon, S}$, then $\mathcal{L}(\lambda)$ is not one-to-one, so it is not bijective.

- If $\lambda \notin \Lambda_{\varepsilon, S}$, then $\mathcal{L}(\lambda)$ is one-to-one. Let us prove that $\mathcal{L}(\lambda)$ is also onto in this case. Let $q=\left(q_{j}\right)_{j=1}^{J_{S}} \in \prod_{j=1}^{J_{S}} L^{2}\left(G_{j}\right)$, and let us build a preimage $v \in D(\mathcal{L})$ of $q$ by $\mathcal{L}(\lambda)$.

Consider first the problems, set in $G_{j}$

$$
\left(\mathcal{P}_{j}^{a}\right) \mid \begin{aligned}
& \text { Find } v_{j}^{a} \in H_{0}^{1}\left(G_{j}\right) \quad \text { such that: } \\
& \varepsilon_{j}\left(d_{\theta}^{2}+\lambda^{2}\right) v_{j}^{a}=q_{j} \quad \text { in } L^{2}\left(G_{j}\right),
\end{aligned}
$$

$j=1 . . J_{S}$. For a given index $j$, the problem $\left(\mathcal{P}_{j}^{a}\right)$ is well-posed within the Fredholm framework. But, the operator $T_{j}:=-d_{\theta}^{2}$ of $L^{2}\left(G_{j}\right)$, with domain $H_{0}^{1}\left(G_{j}\right) \cap H^{2}\left(G_{j}\right)$, is self-adjoint with compact resolvent. Its spectrum is equal to $\left\{k^{2} \pi^{2} /\left(\sigma_{j}-\sigma_{j-1}\right)^{2}, k \in \mathbb{N}^{*}\right\}$. The first (smallest) eigenvalue of $T_{j}, \pi^{2} /\left(\sigma_{j}-\sigma_{j-1}\right)^{2}$, is therefore larger than or equal to 1 because $\sigma_{j}-\sigma_{j-1} \leq \pi$. Then, for $\lambda \in \mathcal{B}(-1 ; 1), \lambda^{2}$ does not belong to the spectrum of $T_{j}$. For the solution of problem $\left(\mathcal{P}_{j}^{a}\right)$ (for $j=1 . . J_{S}$ ), uniqueness follows. Within the Fredholm framework, this shows that, for all $\lambda \in \mathcal{B}(-1 ; 1)$, problem $\left(\mathcal{P}_{j}^{a}\right)$ has one, and only one, soution $v_{j}^{a}, j=1 . . J_{S}$. Moreover, results on the regularity of solutions to elliptic PDEs indicate that $v_{j}^{a} \in H^{2}\left(G_{j}\right) \subset \mathcal{C}^{1}\left(\overline{G_{j}}\right), j=1 . . J_{S}$. 
Next, let us consider the problem

$$
\left(\mathcal{P}^{b}\right) \mid \begin{array}{ll}
\text { Find }\left(v_{j}^{b}\right)_{j=1}^{J_{S}} \in \prod_{j=1}^{J_{S}} H^{1}\left(G_{j}\right) & \text { such that: } \\
\varepsilon_{j}\left(d_{\theta}^{2}+\lambda^{2}\right) v_{j}^{b}=0 & \text { in } G_{j}, j=1 . . J_{S} \\
v_{1}^{b}(0)=v_{J_{S}}^{b}\left(\sigma_{J_{S}}\right)=0 & j=1 . .\left(J_{S}-1\right) \\
v_{j}^{b}\left(\sigma_{j}\right)=v_{j+1}^{b}\left(\sigma_{j}\right) & j=1 . .\left(J_{S}-1\right) \\
\varepsilon_{j} v_{j}^{b \prime}\left(\sigma_{j}\right)-\varepsilon_{j+1} v_{j+1}^{b}{ }^{\prime}\left(\sigma_{j}\right)=-\alpha_{j} & j=\sigma^{\prime}
\end{array}
$$

where the right-hand side $\alpha_{j}$ is equal to $\varepsilon_{j} v_{j}^{a}{ }^{\prime}\left(\sigma_{j}\right)-\varepsilon_{j+1} v_{j+1}^{a}{ }^{\prime}\left(\sigma_{j}\right), j=1 . .\left(J_{S}-1\right)$. If $\left(v_{j}^{b}\right)_{j=1}^{J_{S}}$ solves $\left(\mathcal{P}^{b}\right)$, then the volume PDEs imply that $v_{j}^{b}(\theta)=A_{j} e^{i \lambda \theta}+B_{j} e^{-i \lambda \theta}$ (resp. $v_{j}^{b}(\theta)=A_{j} \theta+B_{j}$ ) for $\lambda \neq 0$ (resp. $\lambda=0$ ). Writing the transmission conditions (there are $2\left(J_{S}-1\right)$ of them), together with the two Dirichlet boundary conditions at 0 and at $\sigma_{J_{S}}$, one builds an algebraic set of $2 J_{S}$ linear equations, with $2 J_{S}$ unknowns. Since $\mathcal{L}(\lambda)$ is one-to-one, it follows that $\left(\mathcal{P}^{b}\right)$ has one, and only one, solution.

Finally, define $v$ by $\left.v\right|_{G_{j}}=v_{j}^{a}+v_{j}^{b}, j=1 . . J_{S}$. It belongs to $D(\mathcal{L})$, and moreover $\mathcal{L}(\lambda) v=q$. This proves that the operator $\mathcal{L}(\lambda)$ is onto.

Next, we state a result on the norm of $\mathcal{L}(\lambda)^{-1}$.

Lemma 4.9. Assume that $\varepsilon_{j}+\varepsilon_{j+1} \neq 0, j=1 . .\left(J_{S}-1\right)$. Consider two real numbers $\alpha, \beta$ with $\alpha<\beta$. Then there exist $\eta_{0}>0$ and $C>0$ independent of $\lambda$ such that,

$$
\sum_{j=1}^{J_{S}}\left\|d_{\theta}^{2} v_{j}\right\|_{L^{2}\left(G_{j}\right)}+|\lambda|\left\|d_{\theta} v\right\|_{L^{2}(G)}+|\lambda|^{2}\|v\|_{L^{2}(G)} \leq C\|\mathcal{L}(\lambda) v\|_{L^{2}(G)},
$$

for all $v \in D(\mathcal{L})$ and all $\lambda \in \mathbb{C}$ such that $|\Im(\lambda)|>\eta_{0}$ and $\alpha<\Re(\lambda)<\beta$.

Proof. This nice result is proved in [16] (lemma 3.6). For the sake of clarity, we reformulate the (slightly modified) proof. Below, $C>0$ designate constant numbers independent of the functions and of $\lambda$. Define the two rectangles $\mathscr{R}_{1}:=\{(s, \theta) \in$ $\mathbb{R}^{2} \mid 1 / 2<s<2$ and $\left.\theta \in G\right\}$ and $\mathscr{R}_{2}:=\left\{(s, \theta) \in \mathbb{R}^{2} \mid 1 / 4<s<4\right.$ and $\left.\theta \in G\right\}$. One has $\mathscr{R}_{1} \subset \mathscr{R}_{2}$. Consider $\zeta$ a smooth cutoff function which only depends on $s$ such that $\zeta=1$ on $\mathscr{R}_{1}$ and $\operatorname{supp} \zeta \subset \mathscr{R}_{2}$. For $v \in D(\mathcal{L})$, define $w:(s, \theta) \mapsto \zeta(s) e^{\lambda s} v(\theta)$. Using the proof of the theorem 4.6 (cf. $\S 4.2$ ), one can first write

$$
\sum_{j=1}^{J_{S}}\left\|w_{j}\right\|_{H^{2}\left(\mathscr{R}_{1} \cap \Gamma_{j}\right)} \leq C\left(\sum_{j=1}^{J_{S}}\left\|\varepsilon_{j} \Delta w_{j}\right\|_{L^{2}\left(\mathscr{R}_{2} \cap \Gamma_{j}\right)}+\|\nabla w\|_{L^{2}\left(\mathscr{R}_{2}\right)}\right) .
$$

On the one hand, in the cartesian coordinate system $(s, \theta)$, one has $\nabla w(s, \theta)=$ $\left(\left(d_{s} \zeta(s)+\lambda \zeta(s)\right) e^{\lambda s} v(\theta), \zeta(s) e^{\lambda s} d_{\theta} v(\theta)\right)$ and $\Delta\left(\zeta(s) e^{\lambda s} v(\theta)\right)(s, \theta)=\zeta(s)\left(d_{\theta}^{2}+\lambda^{2}\right) e^{\lambda s} v(\theta)+$ $2 \lambda d_{s} \zeta(s) e^{\lambda s} v(\theta)+d_{s}^{2} \zeta(s) e^{\lambda s} v(\theta)$. Therefore, one obtains for $|\lambda| \neq 0$

$$
\begin{aligned}
& \sum_{j=1}^{J_{S}}\left\|\varepsilon_{j} \Delta w_{j}\right\|_{L^{2}\left(\mathscr{R}_{2} \cap \Gamma_{j}\right)}+\|\nabla w\|_{L^{2}\left(\mathscr{R}_{2}\right)} \\
\leq & C\left(\|\mathcal{L}(\lambda) v\|_{L^{2}(G)}+\left\|d_{\theta} v\right\|_{L^{2}(G)}+|\lambda|\|v\|_{L^{2}(G)}\right) .
\end{aligned}
$$

Since $d_{s}\left(e^{\lambda s} v(\theta)\right)=\lambda e^{\lambda s} v(\theta) ; \quad d_{\theta}\left(e^{\lambda s} v(\theta)\right)=e^{\lambda s} d_{\theta} v(\theta) ; \quad d_{s}^{2}\left(e^{\lambda s} v(\theta)\right)=\lambda^{2} e^{\lambda s} v(\theta)$; $d_{\theta}^{2}\left(e^{\lambda s} v(\theta)\right)=e^{\lambda s} d_{\theta}^{2} v(\theta) ; \quad d_{s, \theta}^{2}\left(e^{\lambda s} v(\theta)\right)=\lambda e^{\lambda s} d_{\theta} v(\theta)$, one has on the other hand

$$
\sum_{j=1}^{J_{S}}\left\|w_{j}\right\|_{H^{2}\left(\mathscr{R}_{1} \cap \Gamma_{j}\right)} \geq C\left(\sum_{j=1}^{J_{S}}\left\|d_{\theta}^{2} v_{j}\right\|_{L^{2}\left(G_{j}\right)}+|\lambda|\left\|d_{\theta} v\right\|_{L^{2}(G)}+|\lambda|^{2}\|v\|_{L^{2}(G)}\right) .
$$


Plugging (4.10) and (4.11) in (4.9), one finds $\sum_{j=1}^{J_{S}}\left\|d_{\theta}^{2} v_{j}\right\|_{L^{2}\left(G_{j}\right)}+|\lambda|\left\|d_{\theta} v\right\|_{L^{2}(G)}+$ $|\lambda|^{2}\|v\|_{L^{2}(G)} \leq C\left(\|\mathcal{L}(\lambda) v\|_{L^{2}(G)}+\left\|d_{\theta} v\right\|_{L^{2}(G)}+|\lambda|\|v\|_{L^{2}(G)}\right)$. That is equivalent to

$$
\sum_{j=1}^{J_{S}}\left\|d_{\theta}^{2} v_{j}\right\|_{L^{2}\left(G_{j}\right)}+(|\lambda|-C)\left\|d_{\theta} v\right\|_{L^{2}(G)}+|\lambda|(|\lambda|-C)\|v\|_{L^{2}(G)} \leq C\|\mathcal{L}(\lambda) v\|_{L^{2}(G)} .
$$

Taking $\eta_{0}=2 C$, one obtains the result of the lemma.

With the help of the analytic Fredholm theorem, one deduces the

Corollary 4.10. Assume that $\varepsilon_{j}+\varepsilon_{j+1} \neq 0, j=1 . .\left(J_{S}-1\right)$. Then there exist two real numbers $\alpha_{S}, \beta_{S}$ with $0<\alpha_{S}<1,0<\beta_{S}<1$ such that $\left(\mathcal{B}\left(-\alpha_{S} ; \beta_{S}\right) \cap\right.$ $\left.\Lambda_{\varepsilon, S}\right) \subset \mathbb{R} i$. In addition, the cardinality of $\mathcal{B}\left(-\alpha_{S} ; \beta_{S}\right) \cap \Lambda_{\varepsilon, S}$ is finite.

Using the Parseval identity (see the lemma 6.1.4 of [21]), one can now state an isomorphism result between weighted spaces (theorem 3.7 of [16]).

TheOrem 4.11. Assume that $\varepsilon_{j}+\varepsilon_{j+1} \neq 0, j=1 . .\left(J_{S}-1\right)$. Let $\gamma \in \mathbb{R}$ be such that $\{\lambda \in \mathbb{C} \mid \Re(\lambda)=1-\gamma\} \cap_{0} \Lambda_{\varepsilon, S}=\emptyset$. Then, for all $P \in V_{\gamma}^{0}(\Gamma)$, there exists one, and only one, solution $W \in V_{\gamma-1}^{1}(\Gamma)$ to the transmission problem

$$
\begin{array}{l|ll} 
& \text { Find } W \in V_{\gamma-1}^{1}(\Gamma) \text { such that: } \\
\varepsilon_{j} \Delta W_{j}=P_{j} & \text { in } \Gamma_{j}, j=1 . . J_{S} \\
\left.\mathcal{P}_{\text {sector }}^{\gamma}\right) & W_{1}=0 & \text { on } \partial \Gamma_{1} \cap \partial \Gamma \\
& W_{J_{S}}=0 & \text { on } \partial \Gamma_{J_{S}} \cap \partial \Gamma \\
W_{j}-W_{j+1}=0 & \text { on } \partial \Gamma_{j} \cap \partial \Gamma_{j+1}, j=1 . .\left(J_{S}-1\right) \\
\varepsilon_{j} \partial_{\theta} W_{j}-\varepsilon_{j+1} \partial_{\theta} W_{j+1}=0 & \text { on } \partial \Gamma_{j} \cap \partial \Gamma_{j+1}, j=1 . .\left(J_{S}-1\right) .
\end{array}
$$

This solution can be expressed as

$W:(r, \theta) \mapsto W(r, \theta)=\mathcal{M}^{-1}\left(\mathcal{L}(\lambda)^{-1} \hat{H}\right)(r, \theta)=\frac{1}{2 i \pi} \uparrow \int_{\Re(\lambda)=1-\gamma} r^{\lambda} \mathcal{L}(\lambda)^{-1} \hat{H}(\lambda, \theta) d \lambda$

with $H:=r^{2} P$.

Moreover, $W_{j} \in V_{\gamma}^{2}\left(\Gamma_{j}\right), j=1 . . J_{S}$, with the continuity estimate

$$
\|W\|_{V_{\gamma-1}^{1}(\Gamma)}+\sum_{j=1}^{J_{S}}\left\|W_{j}\right\|_{V_{\gamma}^{2}\left(\Gamma_{j}\right)} \leq C\|P\|_{V_{\gamma}^{0}(\Gamma)} .
$$

We already noticed that the right-hand side $p$ that appears in $\left(\mathcal{P}_{\text {sector }}\right)$ belongs to $L^{2}(\Gamma)$ with compact support, so $p \in V_{\gamma}^{0}(\Gamma)$ for all $\gamma \geq 0$.

Definition 4.12. Assume that $\varepsilon_{j}+\varepsilon_{j+1} \neq 0, j=1 . .\left(J_{S}-1\right)$. Let $\gamma \geq 0$ such that $\{\lambda \in \mathbb{C} \mid \Re(\lambda)=1-\gamma\} \cap \Lambda_{\varepsilon, S}=\emptyset$. We denote by $w^{1-\gamma}$ the solution to $\left(\mathcal{P}_{\text {sector }}^{\gamma}\right)$ with right-hand side equal to $p$.

According to previous results, we know that $w^{1-\gamma}$ can be expressed as

$w^{1-\gamma}:(r, \theta) \mapsto w^{1-\gamma}(r, \theta)=\mathcal{M}^{-1}\left(\mathcal{L}(\lambda)^{-1} \hat{h}\right)(r, \theta)=\frac{1}{2 i \pi} \uparrow \int_{\Re(\lambda)=1-\gamma} r^{\lambda} \mathcal{L}(\lambda)^{-1} \hat{h}(\lambda, \theta) d \lambda$.

Moreover, $w^{1-\gamma}$ belongs to $\stackrel{\circ}{V}_{\gamma-1}^{1}(\Gamma)$, and $w_{j}^{1-\gamma} \in V_{\gamma}^{2}\left(\Gamma_{j}\right), j=1 . . J_{S}$.

It turns out that, if $\varepsilon_{j}+\varepsilon_{j+1} \neq 0, j=1 . .\left(J_{S}-1\right)$, then $w^{\beta_{S}}$ (where $\beta_{S}$ appears in corollary 4.10) is well-defined, and moreover it is more regular than $H^{1}$. As a matter 
of fact, one has $w^{\beta_{S}} \in \stackrel{\circ}{V}_{-\beta_{S}}^{1}(\Gamma)$, with $w_{j}^{\beta_{S}} \in V_{1-\beta_{S}}^{2}\left(\Gamma_{j}\right), j=1 . . J_{S}$.

The next step is to prove that $w$ is equal to $w^{\beta_{S}}$. With this result, the extra regularity result will hold, for $S \in \mathcal{S}_{\text {ext }}$. From now on, let us assume that $\varepsilon_{j}+\varepsilon_{j+1} \neq 0$, $j=1 . .\left(J_{S}-1\right)$.

Lemma 4.13. Let $\lambda \in \mathcal{B}(-1 ; 0) \backslash \Lambda_{\varepsilon, S}$. Then $\hat{w}(\lambda, \cdot)$ belongs to $D(\mathcal{L})$, and

$$
\hat{w}(\lambda, \cdot)=\mathcal{L}(\lambda)^{-1} \hat{h}(\lambda, \cdot) .
$$

Proof. As $\Re(\lambda) \leq 0, \hat{w}(\lambda, \cdot)$ is well-defined, and it belongs to $H^{1}(G)$, according to lemma 4.7. But, as $\hat{w}(\lambda, \cdot) \in H^{1}(G)$ is a solution to $\left(\hat{\mathcal{P}}_{\text {sector }}\right)$, it is also an element of $D(\mathcal{L})$. Thanks to lemma 4.8 , there holds $\hat{w}(\lambda, \cdot)=\mathcal{L}(\lambda)^{-1} \hat{h}(\lambda, \cdot)$.

According to theorem 4.11, this proves that

$$
w=w^{-\alpha_{S}}=\frac{1}{2 i \pi} \uparrow \int_{\Re(\lambda)=-\alpha_{S}} r^{\lambda} \mathcal{L}(\lambda)^{-1} \hat{h}(\lambda, \cdot) d \lambda .
$$

LEMmA 4.14. The mapping $\lambda \mapsto \mathcal{L}(\lambda)^{-1} \hat{h}(\lambda, \cdot)$, with values in $D(\mathcal{L})$, defines a meromorphic extension of $\hat{w}(\lambda, \cdot)$ over $\operatorname{int}(\mathcal{B}(-\alpha ; 1))$, for all $\alpha>0$.

Proof. According to the analytic Fredholm theorem, the mapping $\lambda \mapsto \mathcal{L}(\lambda)^{-1}$ is a meromorphic function over $\mathbb{C}$, with values into the set of continuous mappings from $L^{2}(G)$ to $D(\mathcal{L})$. On the other hand, one can prove (see lemma 3.11 of [16]) that $\hat{h}$ is holomorphic with values in $L^{2}(G)$, in $\operatorname{int}(\mathcal{B}(-\alpha ; 1))$, for all $\alpha>0$. Combining these two results leads to the conclusion.

Consider next the contour integral

$\mathcal{C}_{R}:=\left[-R+i \beta_{S} ; R+i \beta_{S}\right] \cup\left[R+i \beta_{S} ; R-i \alpha_{S}\right] \cup\left[R-i \alpha_{S} ;-R-i \alpha_{S}\right] \cup\left[-R-i \alpha_{S} ;-R+i \beta_{S}\right]$

in the complex plane with $R>0$. As $\lambda \mapsto r^{\lambda} \mathcal{L}(\lambda)^{-1} \hat{h}$ is meromorphic in the strip $\mathcal{B}\left(-\alpha_{S} ; \beta_{S}\right)$, Cauchy's theorem indicates that

$$
\begin{aligned}
w^{\beta_{S}}-w & =\frac{1}{2 i \pi} \uparrow \int_{\Re(\lambda)=\beta_{S}} r^{\lambda} \mathcal{L}(\lambda)^{-1} \hat{h}(\lambda, \cdot) d \lambda-\frac{1}{2 i \pi} \uparrow \int_{\Re(\lambda)=-\alpha_{S}} r^{\lambda} \mathcal{L}(\lambda)^{-1} \hat{h}(\lambda, \cdot) d \lambda \\
& =\lim _{R \rightarrow+\infty} \int_{\mathcal{C}_{R}} r^{\lambda} \mathcal{L}(\lambda)^{-1} \hat{h}(\lambda, \cdot) d \lambda \\
& =\sum_{\lambda \in \Lambda_{\varepsilon, S} \cap \mathcal{B}\left(-\alpha_{S} ; \beta_{S}\right)} \operatorname{Res}_{\lambda} r^{\lambda} \mathcal{L}(\lambda)^{-1} \hat{h}(\lambda, \cdot) .
\end{aligned}
$$

Let $\lambda \in \Lambda_{\varepsilon, S} \cap \mathcal{B}\left(-\alpha_{S} ; \beta_{S}\right)$. One can prove (see lemma 5.1.4, theorems 5.4 .1 and 6.1.4 of [21], or alternately [11]), that the residual $\operatorname{Res}_{\lambda} r^{\lambda} \mathcal{L}(\lambda)^{-1} \hat{h}(\lambda, \cdot)$ can be decomposed on a finite set of functions like $r^{\lambda} \sum_{k=0}^{K}(\log r)^{k} \varphi_{k}(\theta) / k$ !, with $\varphi_{k} \in H_{0}^{1}(G)$, for $k=0 . . K$. Now, according to corollary 4.10 , one has $\left(\Lambda_{\varepsilon, S} \cap \mathcal{B}\left(-\alpha_{S} ; \beta_{S}\right)\right) \subset \mathbb{R} i$. As a consequence, for $\lambda \in\left(\Lambda_{\varepsilon, S} \cap \mathcal{B}\left(-\alpha_{S} ; \beta_{S}\right)\right)$, a non-vanishing function $(r, \theta) \mapsto$ $r^{\lambda} \sum_{k=0}^{K}(\log r)^{k} \varphi_{k}(\theta) / k$ ! does not belong to $H^{1}$. Since $w^{\beta_{S}}$ and $w$ both belong locally to $H^{1}$ in a neighborhood of the origin, it follows that necessarily

$$
\sum_{\lambda \in \Lambda_{\varepsilon, S} \cap \mathcal{B}\left(-\alpha_{S} ; \beta_{S}\right)} \operatorname{Res}_{\lambda}^{\lambda} \mathcal{L}(\lambda)^{-1} \hat{h}(\lambda, \cdot)=0 .
$$


Thus, $w=w^{\beta_{S}}$. This implies $w \in V_{-\beta_{S}}^{1}(\Gamma), w_{j} \in V_{1-\beta_{S}}^{2}\left(\Gamma_{j}\right), j=1 . . J_{S}$, with the continuity estimate of theorem 4.11

$$
\|w\|_{V_{-\beta_{S}}^{1}(\Gamma)}+\sum_{j=1}^{J_{S}}\left\|w_{j}\right\|_{V_{1-\beta_{S}}^{2}\left(\Gamma_{j}\right)} \leq C_{1}\|p\|_{V_{1-\beta_{S}}^{0}(\Gamma)}
$$

where the constant $C_{1}$ depends only on $\beta_{S}$.

We are now able to state the regularity result at boundary vertices. Recall that $p$, defined in (4.8), belongs to $L^{2}(\Gamma)$ with compact support, so $p$ is in $V_{1-\beta_{S}}^{0}(\Gamma)\left(\beta_{S}<1\right)$, so one may write

$$
\begin{aligned}
\|p\|_{V_{1-\beta_{S}}^{0}(\Gamma)} & =\left\|\left(x^{2}+y^{2}\right)^{1-\beta_{S}} p\right\|_{L^{2}(\Gamma)} \\
& \leq\left\|\left(x^{2}+y^{2}\right)^{1-\beta_{S}}\right\|_{L^{\infty}(\Gamma)}\|p\|_{L^{2}(\Gamma)} \\
& \leq d_{S}^{2\left(1-\beta_{S}\right)}\|p\|_{L^{2}(\Gamma)} \\
& \leq C_{2}\left(\|f\|_{L^{2}(\Omega)}+\|\nabla u\|_{L^{2}(\Omega)}\right) .
\end{aligned}
$$

This leads to

$$
\|w\|_{V_{-\beta_{S}}^{1}(\Gamma)}+\sum_{j=1}^{J_{S}}\left\|w_{j}\right\|_{V_{1-\beta_{S}}^{2}\left(\Gamma_{j}\right)} \leq C_{1} C_{2}\left(\|f\|_{L^{2}(\Omega)}+\|\nabla u\|_{L^{2}(\Omega)}\right)
$$

which in turns leads to the regularity result for boundary vertices.

Theorem 4.15. Assume that $\varepsilon_{j}+\varepsilon_{j+1} \neq 0, j=1 . .\left(J_{S}-1\right)$. Then, for $\left.\beta_{S} \in\right] 0 ; 1[$ defined in corollary 4.10, there holds

$\|u\|_{V_{-\beta_{S}}^{1}\left(\Omega \cap B\left(S, \frac{d_{S}}{2}\right)\right)}+\sum_{j=1}^{J_{S}}\left\|u_{j}\right\|_{V_{1-\beta_{S}}^{2}\left(\Omega_{j} \cap B\left(S, \frac{d_{S}}{2}\right)\right)} \leq C\left(\|\operatorname{div}(\varepsilon \nabla u)\|_{L^{2}(\Omega)}+\|\nabla u\|_{L^{2}(\Omega)}\right)$

where the constant $C$ is independent of $u$.

4.5. Regularity at interior vertices. Consider an interior vertex $S \in \mathcal{S}_{\text {int }}$. The regularity study around this vertex is very similar to the one which we carried out in the previous subsections. One has to replace the homogeneous Dirichlet boundary conditions at 0 and $\sigma_{J_{S}}=2 \pi$ by two transmission conditions.

Below, we keep the same notations. This time, we define the set $\Lambda_{\varepsilon, S}$ of singular exponents related to the vertex $S$ as the set of complex numbers $\lambda \in \mathbb{C}$ such that there is a non-zero $J_{S}$-tuple $\left(\phi_{\lambda, j}\right)_{j=1}^{J_{S}} \in \prod_{j=1}^{J_{S}} H^{2}\left(G_{j}\right)$ which fulfills the conditions below:

$$
\mid \begin{array}{ll}
\left(\partial_{\theta}^{2}+\lambda^{2}\right) \phi_{\lambda, j}=0 & \text { in } G_{j}, j=1 . . J_{S} \\
\phi_{\lambda, 1}(0)=\phi_{\lambda, J_{S}}(2 \pi) & \\
\varepsilon_{1} \partial_{\theta} \phi_{\lambda, 1}(0)=\varepsilon_{J_{S}} \partial_{\theta} \phi_{\lambda, J_{S}}(2 \pi) & \\
\phi_{\lambda, j}\left(\sigma_{j}\right)=\phi_{\lambda, j+1}\left(\sigma_{j}\right) & j=1 . .\left(J_{S}-1\right) \\
\varepsilon_{j} \partial_{\theta} \phi_{\lambda, j}\left(\sigma_{j}\right)=\varepsilon_{j+1} \partial_{\theta} \phi_{\lambda, j+1}\left(\sigma_{j}\right) & j=1 . .\left(J_{S}-1\right) .
\end{array}
$$

This time, one is led to study the Mellin symbol

$$
\begin{aligned}
& \mathcal{L}(\lambda): \quad D(\mathcal{L}) \quad \longrightarrow \quad \prod_{j=1}^{J_{S}} L^{2}\left(G_{j}\right) \\
& v \longmapsto\left(\varepsilon_{j}\left(d_{\theta}^{2}+\lambda^{2}\right) v_{j}\right)_{j=1}^{J_{S}}
\end{aligned}
$$


where

$$
\begin{aligned}
D(\mathcal{L}):=\left\{v \in H^{1}(G) \mid\right. & v_{j} \in H^{2}\left(G_{j}\right), \varepsilon_{J_{S}} v_{J_{S}}^{\prime}(2 \pi)=\varepsilon_{1} v_{1}^{\prime}(0), \\
& \left.\varepsilon_{j} v_{j}^{\prime}\left(\sigma_{j}\right)=\varepsilon_{j+1} v_{j+1}^{\prime}\left(\sigma_{j}\right), j=1 . .\left(J_{S}-1\right)\right\} .
\end{aligned}
$$

Note that one can prove results similar to lemmas 4.8 and 4.9 and corollary 4.10, using here the above definitions of $D(\mathcal{L})$ and $\mathcal{L}(\lambda)$. Following the lines of proofs for boundary vertices, we have

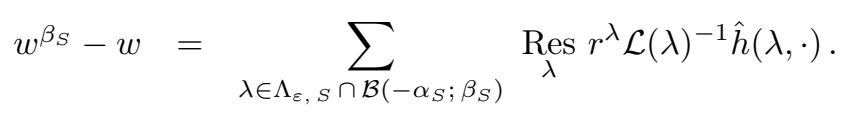

Proceeding as before, because of the lack of homogeneous Dirichlet boundary condition, we can only conclude that

$$
\sum_{\lambda \in \Lambda_{\varepsilon, S} \cap \mathcal{B}\left(-\alpha_{S} ; \beta_{S}\right)} \operatorname{Res}_{\lambda} r^{\lambda} \mathcal{L}(\lambda)^{-1} \hat{h}(\lambda, \cdot)=z_{S},
$$

where $z_{S}$ is a constant. However, this constant is not really annoying, since we are mainly interested in the behavior of $\nabla u$.

We conclude by the result below.

TheOREM 4.16. Assume that $\varepsilon_{j}+\varepsilon_{j+1} \neq 0, j=1 . .\left(J_{S}-1\right)$. Then, there exists a constant number $z_{S}$ such that there holds, with $\left.\beta_{S} \in\right] 0 ; 1[$ defined in corollary 4.10,

$$
\begin{aligned}
& \left\|u+z_{S}\right\|_{V_{-\beta_{S}}^{1}\left(\Omega \cap B\left(S, \frac{d_{S}}{2}\right)\right)}+ \\
& \sum_{j=1}^{J_{S}}\left\|u_{j}+z_{S}\right\|_{V_{1-\beta_{S}}^{2}\left(\Omega_{j} \cap B\left(S, \frac{d_{S}}{2}\right)\right)} \leq C\left(\|\operatorname{div}(\varepsilon \nabla u)\|_{L^{2}(\Omega)}+\|\nabla u\|_{L^{2}(\Omega)}\right)
\end{aligned}
$$

where the constant $C$ is independent of $u$.

4.6. Global regularity. Let us go back to the global regularity problem. We recall that for all $S \in \mathcal{S}, \mathcal{V}_{S}:=\Omega \cap B\left(S, d_{S}\right)$ is a neighborhood of $S$ such that $\overline{\mathcal{V}_{S}} \cap \mathcal{S}=\{S\}$. Consider next an open set $\mathcal{V}_{0} \subset \Omega$ such that $\overline{\mathcal{V}_{0}} \cap \mathcal{S}=\emptyset$ and $\Omega=\mathcal{V}_{0} \cup\left(\cup_{S \in \mathcal{S}} \mathcal{V}_{S}\right)$. For $m \in \mathbb{N}$ and $\gamma \in \mathbb{R}$, let us introduce the functional space

$$
\begin{aligned}
K_{\gamma}^{m}(\Omega):=\left\{v \in \mathcal{D}^{\prime}(\Omega) \mid v\right. & \in H^{m}\left(\mathcal{V}_{0}\right) \text { and } \\
& \left.\forall \alpha \in \mathbb{N},|\alpha| \leq m, \forall S \in \mathcal{S}, r_{S}^{|\alpha|-m+\gamma} \partial_{\boldsymbol{x}}^{\alpha} v \in L^{2}\left(\mathcal{V}_{S}\right)\right\},
\end{aligned}
$$

endowed with the norm

$$
\|v\|_{K_{\gamma}^{m}(\Omega)}:=\left(\|v\|_{H^{m}\left(\mathcal{V}_{0}\right)}^{2}+\sum_{|\alpha| \leq m}\left\|r_{S}^{|\alpha|-m+\gamma} \partial_{\boldsymbol{x}}^{\alpha} v\right\|_{L^{2}\left(\mathcal{V}_{S}\right)}^{2}\right)^{\frac{1}{2}},
$$

where $\left(r_{S}, \theta_{S}\right)$ are the local polar coordinates associated with the vertex $S$.

In addition, for $j=1 . . N, m \in \mathbb{N}$ et $\gamma \in \mathbb{R}$, let us introduce the functional spaces

$$
\begin{aligned}
K_{\gamma}^{m}\left(\Omega_{j}\right):=\left\{v \in \mathcal{D}^{\prime}\left(\Omega_{j}\right) \mid\right. & v \in H^{m}\left(\mathcal{V}_{0} \cap \Omega_{j}\right) \text { and } \\
& \left.\forall \alpha \in \mathbb{N},|\alpha| \leq m, \forall S \in \mathcal{S}, r_{S}^{|\alpha|-m+\gamma} \partial_{x}^{\alpha} v \in L^{2}\left(\mathcal{V}_{S} \cap \Omega_{j}\right)\right\},
\end{aligned}
$$


endowed with the norms

$$
\|v\|_{K_{\gamma}^{m}\left(\Omega_{j}\right)}:=\left(\|v\|_{H^{m}\left(\mathcal{V}_{0} \cap \Omega_{j}\right)}^{2}+\sum_{|\alpha| \leq m}\left\|r_{S}^{|\alpha|-m+\gamma} \partial_{\boldsymbol{x}}^{\alpha} v\right\|_{L^{2}\left(\mathcal{V}_{S} \cap \Omega_{j}\right)}^{2}\right)^{\frac{1}{2}} .
$$

Using a partition of unity, and with the help of theorems 4.1, 4.2, 4.6, 4.15 and 4.16, we can conclude that the following global regularity result holds.

TheOREM 4.17. Assume that, for all couples of subdomains $\left(\Omega_{i}, \Omega_{j}\right)$ of $\Omega$ whose boundary share a common edge, one has $\varepsilon_{i}+\varepsilon_{j} \neq 0$. If one lets $\beta:=\min _{S \in \mathcal{S}} \beta_{S}$, then

$$
\|\nabla u\|_{K_{-\beta}^{0}(\Omega)^{2}}+\sum_{j=1}^{N}\|\nabla u\|_{K_{1-\beta}^{1}\left(\Omega_{j}\right)^{2}} \leq C\left(\|\operatorname{div}(\varepsilon \nabla u)\|_{L^{2}(\Omega)}+\|\nabla u\|_{L^{2}(\Omega)}\right)
$$

where the constant $C$ is independent of $u$.

5. Compact imbedding of $\boldsymbol{X}_{N}(\Omega, \varepsilon)$ into $\boldsymbol{L}^{2}(\Omega)$. We have now at hand all results to prove that the imbedding of $\boldsymbol{X}_{N}(\Omega, \varepsilon)$ into $\boldsymbol{L}^{2}(\Omega)$ is compact.

TheOREM 5.1. Let $\Omega \subset \mathbb{R}^{2}$ be a bounded, open, connected polygonal set of $\mathbb{R}^{2}$, partitioned into $N$ open, polygonal and disjoint subsets $\Omega_{j}$. Assume that, for all couples of subsets $\left(\Omega_{i}, \Omega_{j}\right)$ whose boundary share a common edge, one has $\varepsilon_{i}+\varepsilon_{j} \neq 0$. Then, the imbedding of $\boldsymbol{X}_{N}(\Omega, \varepsilon)$ into $\boldsymbol{L}^{2}(\Omega)$ is compact.

Proof. Let $\left(\boldsymbol{u}_{n}\right)_{n \in \mathbb{N}}$ be a bounded sequence of elements $\boldsymbol{X}_{N}(\Omega, \varepsilon)$. Theorem 3.2 shows that we can associate to $\left(\boldsymbol{u}_{n}\right)_{n \in \mathbb{N}}$ two sequences, respectively $\left(\boldsymbol{u}_{0 n}\right)_{n \in \mathbb{N}} \in$ $\boldsymbol{H}_{N}(\Omega, \varepsilon)^{\mathbb{N}}$ and $\left(\varphi_{n}\right)_{n \in \mathbb{N}} \in D(F)^{\mathbb{N}}$, such that

$$
\boldsymbol{u}_{n}=\boldsymbol{u}_{0 n}+\nabla \varphi_{n}, \forall n \in \mathbb{N}
$$

In addition, the same theorem states that $\left(\boldsymbol{u}_{0 n}\right)_{n \in \mathbb{N}}$ is bounded in $P \boldsymbol{H}^{1}(\Omega, \mathcal{P})$, whereas $\left(\operatorname{div}\left(\varepsilon \nabla \varphi_{n}\right)\right)_{n \in \mathbb{N}}$ and $\left(\nabla \varphi_{n}\right)_{n \in \mathbb{N}}$ are respectively bounded in $L^{2}(\Omega)$, and in $\boldsymbol{L}^{2}(\Omega)$.

But we know that $P \boldsymbol{H}^{1}(\Omega, \mathcal{P})$ is compactly imbedded into $\boldsymbol{L}^{2}(\Omega)$. For instance, we remark that $P \boldsymbol{H}^{1}(\Omega, \mathcal{P}) \subset \cap_{s<\frac{1}{2}} \boldsymbol{H}^{s}(\Omega)$, and that $\boldsymbol{H}^{s}(\Omega)$ is compactly imbedded into $\boldsymbol{L}^{2}(\Omega)$ for any $s>0$, when $\Omega$ is bounded.

One has only to study the sequence $\left(\nabla \varphi_{n}\right)_{n \in \mathbb{N}}$. Denote $\beta:=\min _{S \in \mathcal{S}} \beta_{S}$, where the real numbers $\beta_{S}$ are defined in corollary 4.10. Theorem 4.17 allows us to prove that the sequences $\left(\left.\nabla \varphi_{n}\right|_{\Omega_{j}}\right)_{n \in \mathbb{N}}$ are bounded in $K_{1-\beta}^{1}\left(\Omega_{j}\right)^{2}$, for $j=1 . . N$. Since $\beta>0$, one has $1-\beta<1$, so that $K_{1-\beta}^{1}\left(\Omega_{j}\right)^{2}$ is compactly imbedded into $\boldsymbol{L}^{2}\left(\Omega_{j}\right)$ (cf. lemma 6.2 .1 of [21]), for $j=1 . . N$.

The conclusion follows.

6. Extensions - Case of a curvilinear polygonal domain $\Omega$. Here, we use the definition of a curvilinear polygonal domain given (for instance) in [13].

Definition 6.1. The open subset $\Omega$ of $\mathbb{R}^{2}$ has a $\mathcal{C}^{2}$ curvilinear polygonal boundary $\partial \Omega$ if, for all points a of $\partial \Omega$, there exist $r_{a}>0$ and a diffeomorphism $\chi_{a}$, such that $\chi_{a}$ is a piecewise, $\mathcal{C}^{2}$-diffeomorphism that maps the neighborhood $\mathcal{V}_{a}:=$ $\bar{\Omega} \cap \overline{B\left(a, r_{a}\right)}$ of a to a neighborhood of the origin $O$, included into the plane sector $\Gamma_{a}:=\left\{(r \cos \theta, r \sin \theta) \mid r \geq 0, \theta \in\left[0 ; \omega_{a}\right]\right\}$ of opening $\left.\omega_{a} \in\right] 0 ; 2 \pi[$, a being sent to $O$.

With the help of local maps and going back to the "strict" polygonal boundary case, one is able to prove the result below. 
Theorem 6.2. (Curvilinear Polygon CASe) Let $\Omega \subset \mathbb{R}^{2}$ be a bounded, open, connected subset of $\mathbb{R}^{2}$, with a $\mathcal{C}^{2}$ curvilinear polygonal, connected boundary. Let $\Omega$ be partitioned into $N$ open, disjoint subsets $\Omega_{j}$ with $\mathcal{C}^{2}$ curvilinear polygonal boundaries. Assume that, for all couples of subsets $\left(\Omega_{i}, \Omega_{j}\right)$ whose boundary share a common edge, one has $\varepsilon_{i}+\varepsilon_{j} \neq 0$.

Then, the imbedding of $\boldsymbol{X}_{N}(\Omega, \varepsilon)$ into $\boldsymbol{L}^{2}(\Omega)$ is compact.

7. Case of a non-compact imbedding of $\boldsymbol{X}_{N}(\Omega, \varepsilon)$ into $\boldsymbol{L}^{2}(\Omega)$. To conclude, let us present a negative compact imbedding result. Define $\left.\Omega_{1}:=\right]-1 ; 0[\times] 0 ; 1[$, $\left.\Omega_{2}:=\right] 0 ; 1[\times] 0 ; 1[$ and $\Omega:=]-1 ; 1[\times] 0 ; 1[$ (so that the partition of $\Omega$ is symmetric with respect to the (Oy)-axis). According to theorem 5.1, the imbedding of $\boldsymbol{X}_{N}(\Omega, \varepsilon)$ into $\boldsymbol{L}^{2}(\Omega)$ is compact when $\varepsilon_{1}+\varepsilon_{2} \neq 0$. However, one has the

Proposition 7.1. Assume that $\varepsilon_{1}+\varepsilon_{2}=0$. Then the imbedding of $\boldsymbol{X}_{N}(\Omega, \varepsilon)$ into $\boldsymbol{L}^{2}(\Omega)$ is not compact.

Proof. The idea of the proof can be traced back to proposition 2.7 of [1]. Let $A$ denote the edge $A:=\overline{\Omega_{2}} \cap \overline{\Omega_{1}}$. We recall that $\widetilde{H}^{\frac{1}{2}}(A)$ is the subset of $H^{\frac{1}{2}}(A)$, made up of those elements whose extension by 0 to $\mathbb{R}$ belongs to $H^{\frac{1}{2}}(\mathbb{R})$. Let $\left(g^{k}\right)_{k \in \mathbb{N}}$ be a sequence of elements of $\widetilde{H}^{\frac{1}{2}}(A)$, weakly converging to 0 in $\widetilde{H}^{\frac{1}{2}}(A)$, and such that there is no subsequence that converges strongly in $\widetilde{H}^{\frac{1}{2}}(A)$.

For all $k \in \mathbb{N}$, consider the unique solutions to the problems

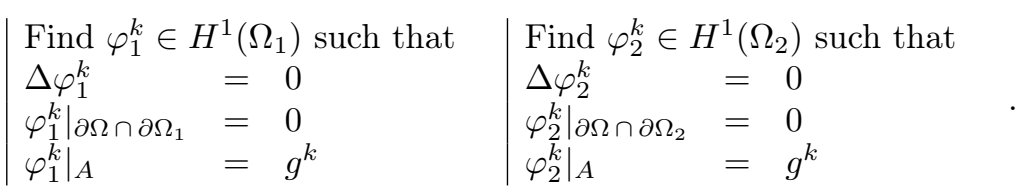

Using the symmetry of the domain, one shows easily that

$$
\varphi_{1}^{k}(x, y)=\varphi_{2}^{k}(-x, y) \text {, a.e. }(x, y) \in \Omega_{1} .
$$

In addition, since the contrast is equal to -1 , the element $\varphi^{k}$ of $H_{0}^{1}(\Omega)$ defined by its restrictions

$$
\varphi^{k}:=\left\{\begin{array}{l}
\varphi_{1}^{k} \text { in } \Omega_{1} \\
\varphi_{2}^{k} \text { in } \Omega_{2}
\end{array}\right.
$$

satisfies $\operatorname{div}\left(\varepsilon \nabla \varphi^{k}\right)=0$.

By construction, the sequence $\left(\varphi^{k}\right)_{k \in \mathbb{N}}$ is bounded in $H^{1}(\Omega)$, and moreover it goes weakly to 0 in $H^{1}(\Omega)$ as the mapping $g^{k} \mapsto \varphi^{k}$ is continuous from $\widetilde{H}^{\frac{1}{2}}(A)$ to $H_{0}^{1}(\Omega)$. However, there exists no subsequence of $\left(\varphi^{k}\right)_{k \in \mathbb{N}}$ that converges strongly to 0 in $H^{1}(\Omega)$ (argue by contradiction, using the continuity of the trace mapping on $A$ ).

Next, define $\boldsymbol{u}^{k}:=\nabla \varphi^{k}$. For all $k \in \mathbb{N}$, there holds curl $\boldsymbol{u}^{k}=0$ and $\operatorname{div}\left(\varepsilon \boldsymbol{u}^{k}\right)=0$. The sequence $\left(\boldsymbol{u}^{k}\right)_{k \in \mathbb{N}}$ is thus bounded in $\boldsymbol{X}_{N}(\Omega, \varepsilon)$, and moreover it converges weakly to 0 in $\boldsymbol{L}^{2}(\Omega)$. On the other hand, one can not extract a subsequence of $\left(\boldsymbol{u}^{k}\right)_{k \in \mathbb{N}}$, that converges strongly (to 0 ) in $\boldsymbol{L}^{2}(\Omega)$. To prove this last result, let us use a contradiction argument: assume there exists a subsequence $\left(\tilde{\boldsymbol{u}}^{k}\right)_{k \in \mathbb{N}}$ of $\left(\boldsymbol{u}^{k}\right)_{k \in \mathbb{N}}$ which converges strongly in $\boldsymbol{L}^{2}(\Omega)$. Then the corresponding subsequence $\left(\tilde{\varphi}^{k}\right)_{k \in \mathbb{N}}$ converges strongly (to 0$)$ in $H^{1}(\Omega)$, hence the contradiction.

REMARK 7.2. This negative imbedding result can obviously be extended to a symmetrically partitioned subset of $\mathbb{R}^{3}$. 
Acknowledgments. The authors express warm thanks for the help and contributions of Anne-Sophie Bonnet-Ben Dhia. They also thank the anonymous referee who suggested a simpler (and more general) framework to carry out the analysis.

\section{REFERENCES}

[1] C. Amrouche, C. Bernardi, M. Dauge, and V. Girault. Vector potentials in three-dimensional non-smooth domains. Math. Meth. Appl. Sci., 21:823-864, 1998.

[2] M. Azaiez, M. Dauge, and Y. Maday. Méthodes spectrales et des éléments finis spectraux. In G. Cohen, editor, Ecole des ondes INRIA : Méthodes numériques d'ordre élevé pour les ondes en régime transitoire, 1994.

[3] C. Bernardi, M. Dauge, and Y. Maday. Compatibilité de traces aux arêtes et coins d'un polyèdre. C. R. Acad. Sci. Paris, Ser. I, 331:679-684, 2000.

[4] M. Sh. Birman and M. Z. Solomyak. $L_{2}$-theory of the Maxwell operator in arbitrary domains. Russ. Math. Surv., 42:75-96, 1987.

[5] M. Sh. Birman and M. Z. Solomyak. Maxwell operator in regions with nonsmooth boundaries. Sib. Math. J., 28:12-24, 1987.

[6] M. Sh. Birman and M. Z. Solomyak. The Weyl asymptotics of the spectrum of the Maxwell operator for domains with a Lipschitz boundary. Vestn. Leningr. Univ., Math., 20:15-21, 1987.

[7] M. Sh. Birman and M. Z. Solomyak. Construction in a piecewise smooth domain of a function of the class $H^{2}$ from the value of the conormal derivative. J. Sov. Math., 49:1128-1136, 1990.

[8] A.-S. Bonnet-Ben Dhia, L. Chesnel, and P. Ciarlet Jr. Optimality of $T$-coercivity for scalar interface problems between dielectrics and metamaterials. submitted.

[9] A.-S. Bonnet-Ben Dhia, P. Ciarlet Jr., and C.M. Zwölf. A new compactness result for electromagnetic waves. application to the transmission problem between dielectrics and metamaterials. Math. Models Meth. App. Sci., 18:1605-1631, 2008.

[10] A.-S. Bonnet-Ben Dhia, P. Ciarlet Jr., and C.M. Zwölf. Time harmonic wave diffraction problems in materials with sign-shifting coefficients. J. Comput. Appl. Math, 234:1912-1919, 2010. Corrigendum J. Comput. Appl. Math., 234:2616, 2010.

[11] A.-S. Bonnet-Ben Dhia, M. Dauge, and K. Ramdani. Analyse spectrale et singularités d'un problème de transmission non coercif. C. R. Acad. Sci. Paris, Ser. I, 328:717-720, 1999.

[12] B.D.F. Casse, H.O. Moser, M. Bahou, J.W. Lee, S. Inglis, and L.K. Jian. Artifical engineering and characterization of micro- and nanoscale electromagnetic metamaterials for the $\mathrm{THz}$ spectral range. Advances in Synchrotron Radiation, 1:1-12, 2008.

[13] M. Costabel and M. Dauge. Weighted regularization of Maxwell equations in polyhedral domains. Numer. Math., 93:239-277, 2002.

[14] M. Costabel, M. Dauge, and S. Nicaise. Singularities of Maxwell interface problems. Math. Mod. Num. Anal., 33:627-649, 1999.

[15] M. Dauge. Elliptic boundary value problems on corner domains. Lecture Notes in Mathematics. Springer-Verlag, Berlin, 1988.

[16] M. Dauge and B. Texier. Problèmes de transmission non coercifs dans des polygones. http://hal.archives-ouvertes.fr/docs/00/56/23/29/PDF/BenjaminT_arxiv.pdf, 2010.

[17] V. Girault and P.-A. Raviart. Finite element methods for Navier-Stokes equations, volume 5 of Springer Series in Computational Mathematics. Springer-Verlag, Berlin, 1986.

[18] P. Grisvard. Elliptic Problems in Nonsmooth Domains. Pitman, London, 1985.

[19] P. Grisvard. Singularities in Boundary Value Problems. RMA 22. Masson, Paris, 1992.

[20] V. A. Kondrat'ev. Boundary-value problems for elliptic equations in domains with conical or angular points. Trans. Moscow Math. Soc., 16:227-313, 1967.

[21] V. A. Kozlov, V. G. Maz'ya, and J. Rossmann. Elliptic Boundary Value Problems in Domains with Point Singularities, volume 52 of Mathematical Surveys and Monographs. AMS, Providence, 1997.

[22] J.-L. Lions and E. Magenes. Problèmes aux limites non homogènes et applications. Dunod, 1968.

[23] P. Monk. Finite element methods for Maxwell's equations. Oxford University Press, New York, 2003.

[24] S. A. Nazarov and B. A. Plamenevsky. Elliptic problems in domains with piecewise smooth boundaries, volume 13 of Expositions in Mathematics. de Gruyter, Berlin, Allemagne, 1994.

[25] B. Prade, J. Y. Vinet, and M. Mysyrowicz. Guided optical waves in planar heterostructures 
with negative dielectric constant. Phys. Rev. B, 44(24):13556-13572, 1991.

[26] D. R. Smith, W. J. Padilla, D. C. Vier, S. C. Nemat-Nasser, and S. Schultz. Composite medium with simultaneously negative permeability and permittivity. Phys. Rev. Lett., 84(18):41844187, 2000.

[27] A. F. Starr, P. M. Rye, D. R. Smith, and S. C. Nemat-Nasser. Fabrication and characterization of a negative-refractive-index composite metamaterial. Phys. Rev. B, 70:113102, 2004.

[28] C. Weber. A local compactness theorem for Maxwell's equations. Math. Meth. Appl. Sci., 2:12-25, 1980. 